\title{
RISK THEORY WITH AFFINE DIVIDEND PAYMENT STRATEGIES
}

\author{
HANSJÖRG ALBRECHER AND ARIAN CANI
}

\begin{abstract}
We consider a classical compound Poisson risk model with affine dividend payments. We illustrate how both by analytical and probabilistic techniques closed-form expressions for the expected discounted dividends until ruin and the Laplace transform of the time to ruin can be derived for exponentially distributed claim amounts. Moreover, numerical examples are given which compare the performance of the proposed strategy to classical barrier strategies and illustrate that such affine strategies can be a noteworthy compromise between profitability and safety in collective risk theory.
\end{abstract}

Dedicated to Robert F. Tichy at the occasion of his 60th birthday.

\section{INTRODUCTION}

The question of how to pay dividends from a surplus process of an insurance portfolio has a long tradition in collective risk theory. The classical criterion to measure the performance of such a dividend strategy is the expected sum of discounted dividend payments over the lifetime of the process, where typically the discount rate is assumed to be positive and constant over time. In this case the optimal strategy is a balance between paying out dividends early (in view of the discounting) and paying dividends later (so that due to the typically positive drift of the process the lifetime (and hence the time span of dividend payments) is prolongated). This criterion was first proposed by de Finetti [11], who proved for a simple random walk model that the optimal strategy is a barrier strategy, that is, dividends are paid out whenever the surplus process exceeds a threshold value (the horizontal dividend barrier), and no dividends are paid out below that level (i.e. the process is reflected at this barrier). Later, Gerber [14] proved that for a Cramér-Lundberg risk process, a so-called band strategy is optimal, which simplifies to a barrier strategy in some particular cases (including the one with exponential claim size distribution). More recently, this stochastic control problem was embedded in modern control theory, which led

Key words and phrases. Cramér-Lundberg risk model; affine dividend payments; hypergeometric functions.

Financial support by the Swiss National Science Foundation Project 200020143889 is gratefully acknowledged. 
to surprisingly challenging mathematical problems (see e.g. Schmidli [24] and Azcue and Muler [7]). The optimal dividend problem was also studied intensively in many different variants, including model variations, transaction costs, as well as other objective functions and constraints, see [3] and [5] for an overview.

One disadvantage of the classical criterion of maximizing the expected sum of discounted dividend payments until ruin is that it focuses on profitability only, and does not consider the lifetime of the controlled process (in particular, under the optimal band strategy, the process will be ruined with probability 1 , and if the barrier is at level 0 , then it is even optimal to pay out all the surplus immediately and get ruined at the occurrence of the first claim payment; we refer to [4] for an overview of the ruin concept and its many mathematical implications). In [28], a variant of the dividend problem was studied, where the objective function is a weighted sum of expected discounted dividend payments until ruin and expected ruin time. It turns out that in such a setting, again a band strategy (respectively, barrier strategy) is optimal, albeit with modified parameters. This approach was then extended to more general models in [19].

The criterion of maximizing the expected sum of discounted dividend payments until ruin may be considered as a somewhat natural target, which also has economic motivation in terms of valuating a company on the basis of this quantity (starting with [16] and later variants within the corporate finance literature). However, if a barrier strategy is optimal, in addition to the solvency aspect mentioned above, this strategy does not pay any dividends whenever the surplus is below the barrier and it pays the maximal feasible amount above the barrier, so that the dividend stream may be very uneven over time. At the same time, empirical research suggests that companies typically strive for a smooth dividend distribution over time with the incentive to gradually move towards a long-term payout ratio (see e.g. Lintner [18] for a pioneering study on this topic). This goes in line with the observation that dividend payments in practice often adjust to changes in earnings only slowly (indicating that the management exhibits some reluctance to either increase or decrease established dividend levels unless there is sufficient confidence that the new levels are justified for the future, not the least to avoid psychological effects entailed by dividend reductions), see also Brav et al. [8].

In view of these aspects, in this paper we propose a dividend strategy that secures a continuous dividend payment stream, the rate of which is adjusted according to the present surplus value in an affine way. We will study such a strategy for a compound Poisson surplus model. Our approach is in part inspired by Avanzi and Wong [6] who studied a related strategy for a diffusion process and also gave an extensive numerical 
study of its performance. Mathematically, our model in the Cramér-Lundberg framework will lead to an Ornstein-Uhlenbeck process driven by the compound Poisson subordinator. For such a setup we will derive equations for the expected discounted dividend payments until ruin as well as for the Laplace transform of the time of ruin. These equations turn out to be challenging in their own right, and various different approaches to solve them will lead to interesting relations between special functions of hypergeometric type. ${ }^{1}$

An interesting consequence of the numerical results at the end of the paper is that utilizing such an affine dividend strategy leads to almost the same performance as the barrier strategy in terms of expected sum of discounted dividend payments, but has - in many different parameter settings - a considerably longer lifetime. Consequently, in view of a compromise between profitability and safety, such an affine strategy is certainly an interesting alternative. In fact, such a strategy is known to be optimal in a somewhat different context of linear quadratic optimal control problems, where quadratic deviations of a target 'dividend' rate are punished in the objective function, see Steffensen [27] for an application in the control of pension funds and Parlar [22] for a model in forest management systems.

The rest of the paper is organized as follows. In Section 2, we introduce the model and discuss some basic properties. Section 3 then derives the integro-differential equation for the expected discounted dividend payments and studies its solution for the case of exponentially distributed claim amounts. In Section 4 we pursue an alternative approach for the solution of the latter equation via Laplace transforms, leading to a rather intricate study of certain special functions and suggesting an identity that seems to be new and non-obvious. In Section 5 we adapt the calculations of Section 3 to study the Laplace transform of the time to ruin. In order to retrieve a concrete formula for the expected ruin time from the Laplace transform, we then employ an approach based on digamma functions and another one based on Kampé de Fériet functions. Section 6 gives a simple and intuitive probabilistic view to connect the quantities of Sections 3 and 5. Finally, Section 7 provides detailed numerical illustrations to test the proposed strategy and determines optimal parameters. The results are then compared to the optimal barrier strategies showing that affine strategies can be a competitive alternative to barrier strategies when paying dividends.

\footnotetext{
${ }^{1}$ In this way, a practically motivated question of insurance risk theory leads to non-trivial mathematical problems and relations, a connection which is also in the tradition of Robert Tichy's work, to whom this paper is dedicated. For the application of Quasi-Monte Carlo results to risk theory by Robert Tichy, see e.g. [29, 2].
} 
2. THE MODEL

In the classical Cramér-Lundberg risk model, the surplus process of an insurance company $\left(R_{t}\right)_{t \geq 0}$ is described by

$$
R_{t}=x+c t-\sum_{i=1}^{N_{t}} Y_{i}, \quad t \geq 0,
$$

where $x=R_{0}$ is the initial capital, $c>0$ is the constant premium rate and the claims $\left\{Y_{i}\right\}_{i \in \mathbb{N}}$ are a sequence of independent and identically distributed positive random variables with distribution function $F_{Y}$, bounded density $f_{Y}$ and finite mean $\mu$. The number of claims up to time $t \geq 0$ is assumed to be a homogeneous Poisson process $N_{t}$ with intensity $\lambda>0$, independent of $\left\{Y_{i}\right\}_{i \in \mathbb{N}}$.

Let $D_{t}$ denote the accumulated dividends paid up to time $t$, so $X_{t}:=R_{t}-D_{t}$ is the surplus process after dividend payments. Assume now that dividends are paid according to an affine strategy, i.e.

$$
d D_{t}=\left(q X_{t}+\beta\right) d t
$$

where $q>0$ is a fixed proportionality constant and $0 \leq \beta \leq c$ is a constant rate. Then

$$
d X_{t}=\left(c-\left(q X_{t}+\beta\right)\right) d t-d S_{t}
$$

which identifies $X_{t}$ as a Lévy-driven Ornstein-Uhlenbeck process (in the present case, the driving Lévy process is the compound Poisson process $S_{t}=\sum_{i=1}^{N_{t}} Y_{i}$ ). The unique solution to (3) is given in terms of the stochastic integral

$$
X_{t}=\frac{c-\beta}{q}+\left(x-\frac{c-\beta}{q}\right) e^{-q t}-\int_{0}^{t} e^{-q(t-u)} d S_{u},
$$

i.e.

$$
X_{t}=\frac{c-\beta}{q}+\left(x-\frac{c-\beta}{q}\right) e^{-q t}-\sum_{i=1}^{N_{t}} e^{-q\left(t-T_{i}\right)} Y_{i},
$$

which embeds $X_{t}$ into the class of shot-noise processes. One sees that the process $X_{t}$ behaves like an exponentially decaying function between the claim occurrences, and the influence of past claims on the value of $X_{t}$ also decays exponentially in time (see Figure 1 for a sample path of $X_{t}$ ). 


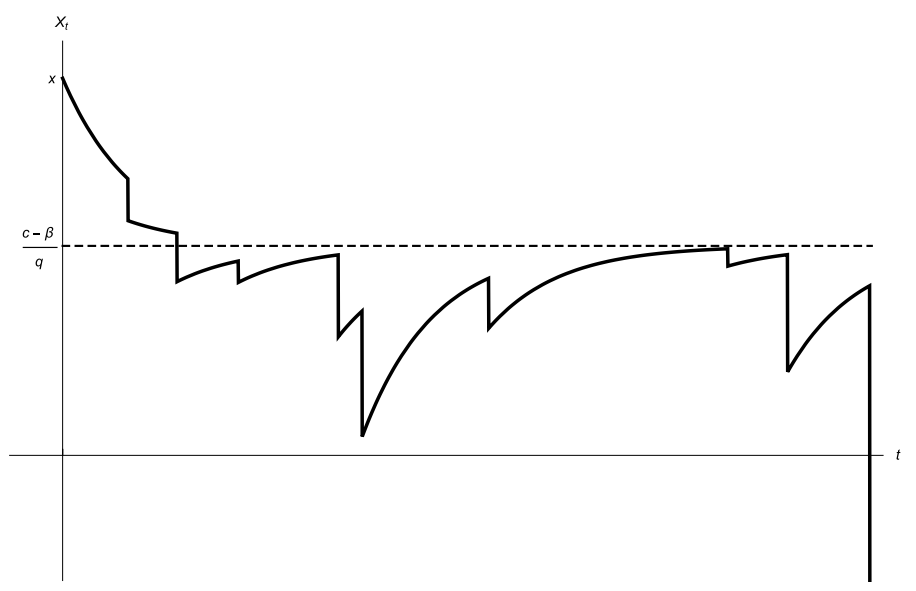

Figure 1: Sample path of $X_{t}$

Let

$$
\tau_{x}:=\inf \left\{t \geq 0: X_{t}<0 \mid X_{0}=x\right\}
$$

denote the time of ruin of $X_{t}$ and note that $P\left(\tau_{x}<\infty\right)=1$ for all $x \geq 0$ (i.e. ruin is certain). The latter holds true, since the process $X_{t}$ is upper-bounded by $\max \{x,(c-\beta) / q\}$ (above $(c-\beta) / q$ there is a negative drift down to this level and below it is bounded by this level).

If $X_{t}$ is not stopped at ruin, then as $t \rightarrow \infty$

$$
X_{t} \stackrel{\text { a.s. }}{\longrightarrow} X_{\infty}:=\frac{c-\beta}{q}-\int_{0}^{\infty} e^{-q u} d S_{u},
$$

see e.g. [23]. If the claim sizes $Y_{i}$ are $\operatorname{Exp}(\alpha)$-distributed, then the self-decomposable limit random variable $X_{\infty}$ simplifies further to a shifted Gamma random variable $X_{\infty}=(c-\beta) / q-Z$ with $Z \sim \Gamma(\lambda / q, \alpha)$, see also [13, 9, 25].

\section{EXPECTED DisCOUNTED DIVIDEND PAYMENTS}

We are now interested in the expected value of the sum of the discounted dividend payments up to the time of ruin

$$
V(x):=\mathbb{E}_{x}\left[\int_{0}^{\tau_{x}} e^{-\delta t}\left(q X_{t}+\beta\right) d t\right]
$$

where $\delta \geq 0$ is a force of interest for valuation. Let us first consider some elementary, but general properties of the function $V(x)$ regarding bounds and growth rate. 
Proposition 3.1. For $x \geq 0$, the function $V(x)$ satisfies the following bounds:

$$
\underline{l}+\frac{q x}{q+\delta} \leq V(x) \leq \bar{l}+\frac{q x}{q+\delta},
$$

where $\underline{l}=\frac{\lambda(q(c-\lambda \mu)+\delta \beta)}{(q+\delta)(\delta+\lambda)}$ and $\bar{l}=\frac{c q+\delta \beta}{\delta(q+\delta)}$.

Proof. For any $t \geq 0$, the process $X_{t}$ in (3) satisfies $X_{t} \leq\left(x-\frac{c-\beta}{q}\right) e^{-q t}+\frac{c-\beta}{q}:=\tilde{X}_{t}$. Then, clearly,

$$
V(x) \leq \mathbb{E}_{x}\left[\int_{0}^{\infty} e^{-\delta t}\left(q \tilde{X}_{t}+\beta\right) d t\right]=\frac{q x-c+\beta}{q+\delta}+\frac{c}{\delta},
$$

which yields the upper bound.

For the lower bound, define $h(x):=\frac{q x}{q+\delta} 1_{\{x \geq 0\}}$ and let $M$ be an operator acting on $h$ defined as

$$
(M h)(x):=\mathcal{L} h(x)-\delta h(x)+q x+\beta,
$$

for $x \geq 0$, where $\mathcal{L} h(x):=(c-(q x+\beta)) h^{\prime}(x)+\lambda\left(\int_{0}^{x} h(x-y) d F_{Y}(y)-h(x)\right)$ is the infinitesimal generator of the process (3). More concretely, (8) can be rewritten as

$$
\begin{aligned}
(M h)(x) & =(c-(q x+\beta)) \frac{q}{q+\delta}+\lambda\left(\int_{0}^{x} \frac{q(x-y)}{q+\delta} d F_{Y}(y)-\frac{q x}{q+\delta}\right)-\frac{\delta q x}{q+\delta}+q x+\beta, \\
& =\frac{c q+\delta \beta}{q+\delta}+\frac{\lambda q x}{q+\delta}\left(F_{Y}(x)-1\right)-\frac{\lambda q}{q+\delta} \int_{0}^{x} y d F_{Y}(y) .
\end{aligned}
$$

Observe that $(M h)^{\prime}(x)=\frac{\lambda q}{q+\delta}\left(F_{Y}(x)-1\right) \leq 0$ with boundary values $(M h)(0)=$ $\frac{c q+\delta \beta}{q+\delta}>0$ and $\lim _{x \rightarrow \infty}(M h)(x)=\frac{q(c-\lambda \mu)+\delta \beta}{q+\delta}>0$. Thus, $(M h)(x)$ is strictly positive and monotone decreasing, bounded from below by $\frac{q(c-\lambda \mu)+\delta \beta}{q+\delta}$.

In view of the Dynkin formula applied to the function $e^{-\delta t} h\left(X_{t}\right)$, the process

$$
e^{-\delta t} h\left(X_{t}\right)-h(x)-\int_{0}^{t} e^{-\delta s}\left[\mathcal{L} h\left(X_{s}\right)-\delta h\left(X_{s}\right)\right] d s
$$

is a zero-expectation martingale. Bearing in mind that the stopped process $X_{t \wedge \tau}$ is also a martingale, we obtain

$$
\mathbb{E}_{x}\left(e^{-\delta t \wedge \tau} h\left(X_{t \wedge \tau}\right)\right)=h(x)+\mathbb{E}_{x}\left(\int_{0}^{t \wedge \tau} e^{-\delta s}\left[\mathcal{L} h\left(X_{s}\right)-\delta h\left(X_{s}\right)\right] d s\right) .
$$

From the properties of $M$, we get that the integrand on the right-hand side is bounded from below by $-\left(q X_{s}+\beta\right)+\frac{q(c-\lambda \mu)+\delta \beta}{q+\delta}$. Furthermore, since $h\left(X_{t \wedge \tau}\right)$ is linearly bounded 
in $t$, an application of the monotone convergence theorem implies that as $t \rightarrow \infty$, the right-hand side converges to 0 . Combining the above and rearranging terms yields

$$
\begin{aligned}
\mathbb{E}_{x}\left[\int_{0}^{\tau} e^{-\delta s}\left(q X_{s}+\beta\right) d s\right] & \geq h(x)+\mathbb{E}_{x}\left[\int_{0}^{\tau} e^{-\delta s} \frac{q(c-\lambda \mu)+\delta \beta}{q+\delta} d s\right] \\
& \geq h(x)+\mathbb{E}_{x}\left[\int_{0}^{T_{1}} e^{-\delta s} \frac{q(c-\lambda \mu)+\delta \beta}{q+\delta} d s\right] \\
& =h(x)+\frac{\lambda(q(c-\lambda \mu)+\delta \beta)}{(q+\delta)(\delta+\lambda)}
\end{aligned}
$$

which gives the result.

Proposition 3.2. For $0 \leq y<x$ and $\overline{f_{Y}}:=\max _{x} f_{Y}(x)<\infty$, the following inequality holds

$$
\frac{\lambda q(x-y)}{q+\lambda+\delta} \leq V(x)-V(y) \leq \frac{q(x-y)}{q+\delta}\left(1+\left(x-y+\frac{c}{\delta}+\frac{\beta}{q}\right) \overline{f_{Y}}\right) .
$$

Proof. Let $0 \leq y<x$ and let $X_{t}^{y}$ and $X_{t}^{x}$ be the processes in (4) started in $y$ and $x$ with respective times of ruin $\tau_{y}$ and $\tau_{x}$. Additionally, define $\mathcal{M}=\left\{\omega \in \Omega \mid \tau_{x}(\omega)=\tau_{y}(\omega)\right\}$ and denote by $\mathcal{M}^{c}$ its complementary set. A pathwise comparison of both processes on $\mathcal{M}$ gives $X_{t}^{x}(\omega)-X_{t}^{y}(\omega)=(x-y) e^{-q t}$. We have

$$
\begin{aligned}
V(x)-V(y) & =\mathbb{E}\left[\int_{0}^{\tau_{y}} e^{-\delta t} q X_{t}^{x} d t\right]-\mathbb{E}\left[\int_{0}^{\tau_{y}} e^{-\delta t} q X_{t}^{y} d t\right]+\mathbb{E}\left[1_{\mathcal{M}^{c}} \int_{\tau_{y}}^{\tau_{x}} e^{-\delta t}\left(q X_{t}^{x}+\beta\right) d t\right], \\
& =\mathbb{E}\left[\int_{0}^{\tau_{y}} e^{-(q+\delta) t} q(x-y) d t\right]+\mathbb{E}\left[1_{\mathcal{M}^{c}} \int_{\tau_{y}}^{\tau_{x}} e^{-\delta t}\left(q X_{t}^{x}+\beta\right) d t\right], \\
& \geq \mathbb{E}\left[\int_{0}^{T_{1}} e^{-(q+\delta) t} q(x-y) d t\right] \\
& =\frac{\lambda q(x-y)}{q+\lambda+\delta} .
\end{aligned}
$$

For the reverse direction, we can write

$$
\begin{aligned}
V(x)-V(y) & =\mathbb{E}\left[\int_{0}^{\tau_{y}} e^{-(q+\delta) t} q(x-y) d t\right]+\mathbb{E}\left[1_{\mathcal{M}^{c}} \int_{\tau_{y}}^{\tau_{x}} e^{-\delta t} X_{t}^{x} d t\right], \\
& \leq \int_{0}^{\infty} e^{-(q+\delta) t} q(x-y) d t+V(x-y) \mathbb{E}\left[1_{\mathcal{M}^{c}}\right]
\end{aligned}
$$

The last inequality follows from the a.s. finiteness of $\tau_{y}$ in the first integral combined with the strong Markov property of the process $X^{x}$ and observing that on $\mathcal{M}^{c}$, 
$X_{\tau_{y}}^{x}(\omega) \leq(x-y) e^{-q \tau_{y}(\omega)} \leq x-y$ in the second integral. By definition, $\mathcal{M}^{c}$ comprises all paths $\omega$ such that $\tau_{x}(\omega)>\tau_{y}(\omega)$, therefore $\mathbb{E}\left[1_{\mathcal{M}^{c}}\right]=P\left(\tau_{x}>\tau_{y}\right)$. Writing $X_{\tau_{y-}}^{y}$ for the surplus immediately prior to ruin of the surplus started in $y$ and conditioning on the latter leads to

$$
\begin{aligned}
P\left(\tau_{x}>\tau_{y}\right) & =\int_{0}^{\max \left(y, \frac{c-\beta}{q}\right)} P\left(\tau_{x}>\tau_{y} \mid X_{\tau_{y-}}^{y}=z\right) P\left(X_{\tau_{y-}}^{y} \in d z\right) \\
& =\int_{0}^{\max \left(y, \frac{c-\beta}{q}\right)} P(z<Y \leq z+x-y) P\left(X_{\tau_{y-}}^{y} \in d z\right), \\
& =\int_{0}^{\max \left(y, \frac{c-\beta}{q}\right)} \int_{z}^{z+x-y} f_{Y}(w) d w P\left(X_{\tau_{y-}}^{y} \in d z\right) \leq(x-y) \overline{f_{Y}}
\end{aligned}
$$

Substituting the last result in (9) and explicitly evaluating the first integral in the aforementioned expression gives

$$
V(x)-V(y) \leq \frac{q(x-y)}{q+\delta}+V(x-y)(x-y) \overline{f_{Y}} .
$$

Combining this with the upper bound obtained in Proposition 3.1 establishes the result.

Hence $V(x)$ is locally Lipschitz and differentiable almost everywhere. If the derivative exists, then using the typical infinitesimal generator arguments for $X_{t}$ and in view of $(7)$, one gets that $V(x)$ is characterized as a solution to the integro-differential equation (IDE)

$$
(c-(q x+\beta)) V^{\prime}(x)-(\lambda+\delta) V(x)+\lambda \int_{0}^{x} V(x-y) d F_{Y}(y)=-(q x+\beta), \quad x \geq 0 .
$$

3.1. Constructing an exact solution for exponential claims. We now assume that the claims are exponentially distributed with rate $\alpha>0$. Then, applying the operator $\left(\frac{d}{d x}+\alpha\right)$ to both sides of (10) leads to the second-order differential equation

$$
\begin{aligned}
(c-(q x+\beta)) V^{\prime \prime}(x)+[\alpha(c-(q x+\beta))-(q+\lambda+\delta)] & V^{\prime}(x)-\alpha \delta V(x) \\
& =-q(1+\alpha x)-\alpha \beta .
\end{aligned}
$$

Let $V_{h}$ be the solution to the related homogeneous differential equation of (11). Choosing $f(z):=V_{h}(x)$ associated to the change of variable $z:=z(x)=\frac{\alpha(c-(q x+\beta))}{q}$ produces Kummer's confluent hypergeometric equation

$$
z f^{\prime \prime}(z)+(b-z) f^{\prime}(z)-a f(z)=0, \quad z \leq \frac{\alpha(c-\beta)}{q},
$$


with parameters

$$
a=\frac{\delta}{q}, \quad b=1+\frac{\lambda+\delta}{q},
$$

which has a regular singular point at $z=0$ and an irregular singular point at $z=-\infty$ (which in the original coordinates correspond to $x=(c-\beta) / q \geq 0$ and $x=\infty$, respectively). This gives

$$
\begin{cases}A_{1} M\left(\frac{\delta}{q}, 1+\frac{\lambda+\delta}{q}, z(x)\right)+A_{2} U\left(\frac{\delta}{q}, 1+\frac{\lambda+\delta}{q}, z(x)\right), & 0 \leq x \leq \frac{c-\beta}{q}, \\ A_{3} M\left(\frac{\delta}{q}, 1+\frac{\lambda+\delta}{q}, z(x)\right)+A_{4} e^{z(x)} U\left(1+\frac{\lambda}{q}, 1+\frac{\lambda+\delta}{q},-z(x)\right), & x>\frac{c-\beta}{q},\end{cases}
$$

for arbitrary constants $A_{i}, i=1, \ldots 4$. Here

$$
M(a, b, z)={ }_{1} F_{1}(a, b, z)=\sum_{n=0}^{\infty} \frac{(a)_{n}}{(b)_{n}} \frac{z^{n}}{n !}
$$

denotes the Kummer confluent hypergeometric function with the Pochhammer symbol $(a)_{n}=\Gamma(a+n) / \Gamma(n)$, and

$$
U(a, b, z)= \begin{cases}\frac{\Gamma(1-b)}{\Gamma(1+a-b)} M(a, b, z)+\frac{\Gamma(b-1)}{\Gamma(a)} z^{1-b} M(1+a-b, 2-b, z) & b \notin \mathbb{Z}, \\ \lim _{\theta \rightarrow b} U(a, \theta, z) & b \in \mathbb{Z},\end{cases}
$$

is Tricomi's confluent hypergeometric function. The piecewise construction of $V_{h}$ originates from the fact that Tricomi's function $U(a, b, z)$ is in general complex-valued when its argument $z$ is negative, that is, when $x>(c-\beta) / q$. Since we are looking for a real-valued solution $V$ over the entire domain $x \geq 0$, another independent pair of solutions to (12), here, $M(a, b, z)$ and $e^{z} U(b-a, b,-z)$ needs to be chosen for $z<0$, namely, $x>(c-\beta) / q$.

The general solution to (11) can then be written as

$$
V(x)=V_{h}(x)+V_{p}(x),
$$

where $V_{p}(x)$ is a particular solution to (11). Looking for a form $V_{p}(x)=A x+B$, one finds

$$
V_{p}(x)=\frac{1}{q+\delta}\left(q x+\beta+\frac{q}{\delta}\left(c-\frac{\lambda}{\alpha}\right)\right), \quad x \geq 0 .
$$

To determine the constant coefficients $A_{i}, i=1, \ldots 4$, we first investigate the components of $V_{h}$ involving the Tricomi function $U$. For $a=\delta / q$ and $b=1+\frac{\lambda+\delta}{q}>1$, $U(a, b, z)$ is singular at $z=0$. Linear boundedness of $V$ established in Proposition 
3.1 then leads to the requirement $A_{2}=0$. Next, we focus on $A_{4}$ : one has (cf. Olver [21])

$$
\lim _{z \rightarrow 0} U(a, b, z)= \begin{cases}\frac{\Gamma(b-1)}{\Gamma(a)} z^{1-b}+\frac{\Gamma(1-b)}{\Gamma(a-b+1)}+\mathcal{O}\left(z^{2-\Re(b)}\right) & \text { if } 1 \leq \Re(b)<2, \\ \frac{1}{\Gamma(a)} z^{-1}+\mathcal{O}(\log z) & \text { if } b=2, \\ \frac{\Gamma(b-1)}{\Gamma(a)} z^{1-b}+\frac{\Gamma(1-b)}{\Gamma(a-b+1)}+\mathcal{O}\left(z^{2-\Re(b)}\right) & \text { if } \Re(b) \geq 2, b \neq 2 .\end{cases}
$$

In the original coordinates, this translates to

$$
\begin{aligned}
\lim _{x \rightarrow \frac{c-\beta}{q}+} e^{z(x)} U\left(1+\frac{\lambda}{q}, 1+\frac{\lambda+\delta}{q},-z(x)\right)= \\
\begin{cases}\frac{\Gamma\left(\frac{\lambda+\delta}{q}\right)}{\Gamma\left(1+\frac{\lambda}{q}\right)}(-z(x))^{-\frac{\lambda+\delta}{q}}+\frac{\Gamma\left(-\frac{\lambda+\delta}{q}\right)}{\Gamma\left(1-\frac{\delta}{q}\right)}++\mathcal{O}\left(x-\frac{c-\beta}{q}\right)^{1-\frac{\lambda+\delta}{q}} & \text { if } \frac{\lambda+\delta}{q}<1, \\
\frac{1}{\Gamma\left(1+\frac{\lambda}{q}\right)}(-z(x))^{-1}+\mathcal{O}\left(\log \left(x-\frac{c-\beta}{q}\right)\right) & \text { if } \frac{\lambda+\delta}{q}=1, \\
\frac{\Gamma\left(\frac{\lambda+\delta}{q}\right)}{\Gamma\left(1+\frac{\lambda}{q}\right)}(-z(x))^{-\frac{\lambda+\delta}{q}}+\frac{\Gamma\left(-\frac{\lambda+\delta}{q}\right)}{\Gamma\left(1-\frac{\delta}{q}\right)}+\mathcal{O}\left(x-\frac{c-\beta}{q}\right)^{1-\frac{\lambda+\delta}{q}} & \text { if } \frac{\lambda+\delta}{q}>1 .\end{cases}
\end{aligned}
$$

The latter expression is unbounded for all choices of $(\lambda+\delta) / q$, so that by the linear boundedness of $V$ we can also conclude $A_{4}=0$. On the other hand, the Kummer function $M$ is analytic over the entire domain $x \geq 0$.

Next, the constant $A_{1}$ is determined by setting $x=0$ in (10) which yields $(c-$ $\beta) V^{\prime}(0)=(\lambda+\delta) V(0)=-\beta$. Using the differentiation property

$$
\frac{d}{d z} M(a, b, z)=\frac{a}{b} M(a+1, b+1, z)
$$

(see [1]), this translates into

$$
\begin{gathered}
(c-\beta)\left[\frac{-\alpha \delta}{q+\lambda+\delta} A_{1} M\left(1+\frac{\delta}{q}, 2+\frac{\lambda+\delta}{q}, z(0)\right)+\frac{q}{q+\delta}\right]+(\lambda+\delta) A_{1} M\left(\frac{\delta}{q}, 1+\frac{\lambda+\delta}{q}, z(0)\right) \\
+(\lambda+\delta)\left[\frac{q}{\alpha \delta(q+\delta)}(\alpha(c-\beta)-(q+\lambda+\delta))+\frac{q+\alpha \beta}{\alpha \delta}\right]=-\beta .
\end{gathered}
$$

Solving for $A_{1}$ gives

$$
A_{1}=\frac{\beta+\frac{q(c-\beta)}{q+\delta}-(\lambda+\delta)\left[\frac{q}{\alpha \delta(q+\delta)}(\alpha(c-\beta)-(q+\lambda+\delta))+\frac{q+\alpha \beta}{\alpha \delta}\right]}{\frac{\alpha \delta(c-\beta)}{q+\lambda+\delta} M\left(1+\frac{\delta}{q}, 2+\frac{\lambda+\delta}{q}, z(0)\right)+(\lambda+\delta) M\left(\frac{\delta}{q}, 1+\frac{\lambda+\delta}{q}, z(0)\right)} .
$$


Finally, by the continuity of $V$ at $x=(c-\beta) / q$ (which follows from Proposition 3.2), we get $A_{3}=A_{1}$, so that we arrive at the following result.

Proposition 3.3. For any $x \geq 0$, the sum of the expected discounted dividend payments up to the time of ruin in a Cramér-Lundberg model with affine dividend strategy (2) and $\operatorname{Exp}(\alpha)$-distributed claims is given by

$V(x)=\frac{\beta+\frac{q(c-\beta)}{q+\delta}-\frac{\lambda+\delta}{q+\delta}\left(\beta+\frac{q}{\delta}\left(c-\frac{\lambda}{\alpha}\right)\right)}{\frac{\alpha \delta(c-\beta)}{q+\lambda+\delta} M\left(1+\frac{\delta}{q}, 2+\frac{\lambda+\delta}{q}, z(0)\right)+(\lambda+\delta) M\left(\frac{\delta}{q}, 1+\frac{\lambda+\delta}{q}, z(0)\right)} M\left(\frac{\delta}{q}, 1+\frac{\lambda+\delta}{q}, z(x)\right)$

$$
+\frac{1}{q+\delta}\left(q x+\beta+\frac{q}{\delta}\left(c-\frac{\lambda}{\alpha}\right)\right)
$$

where $z(x)=\frac{\alpha(c-(q x+\beta))}{q}$.

Remark 3.1. For $q \rightarrow \infty$ (i.e. infinite dividend rate), $V(x)$ in (17) tends to $x+\frac{c}{\lambda+\delta}$. Note that an infinite rate $q$ instantaneously drives the process $X_{t}$ to 0 implying an immediate lump sum dividend payment of size $x$. From then on, all incoming premium at rate $c$ is immediately paid out as dividends (of which a magnitude $c-\beta$ is due to the proportional factor and $\beta$ is due to the constant part), and the process $X_{t}$ is continuously pushed back towards 0 . The first claim will then lead to ruin and stops the dividend payments. That is, $q \rightarrow \infty$ corresponds to a horizontal dividend barrier strategy with barrier $b=0$.

\section{A LAPlace transform APPROACH}

The structure of equation (10) suggests that a Laplace transform approach could in general also be a feasible tool to determine $V$. Indeed, denote by

$$
\widetilde{V}(s):=\int_{0}^{\infty} e^{-s x} V(x) d x, \quad \widetilde{f_{Y}}(s):=\int_{0}^{\infty} e^{-s x} f_{Y}(x) d x
$$

the corresponding Laplace transforms. Then (10) turns into a first-order differential equation for $\widetilde{V}(s)$ :

$$
\widetilde{V}^{\prime}(s)=\widetilde{V}(s) \frac{(\beta-c) s-q+\lambda+\delta-\lambda \widetilde{f_{Y}}(s)}{q s}+\frac{(c-\beta) V(0)-\frac{q}{s^{2}}-\frac{\beta}{s}}{q s} .
$$

It has the solution

$$
\begin{aligned}
\widetilde{V}(s)=e^{-\frac{(c-\beta)}{q} s} s^{\frac{\lambda+\delta}{q}-1} & e^{-\frac{\lambda}{q} \int \frac{\widetilde{f_{Y}}(s)}{s} d s} . \\
& \left(\int \frac{(c-\beta) V(0)-\frac{q}{s^{2}}-\frac{\beta}{s}}{q s} e^{\frac{(c-\beta)}{q} s} s^{1-\frac{\lambda+\delta}{q}} e^{\frac{\lambda}{q} \int \frac{\widetilde{f_{Y}}(s)}{s} d s} d s+C\right)
\end{aligned}
$$


for some constant $C$. In addition to the algebraic manipulations required in the Laplace transform domain, the inversion of $\widetilde{V}(s)$ is another intricate problem, see Section 4.1.

4.1. Exponential claims. It is instructive to see how for exponential claims with rate $\alpha$ the above expression simplifies to the explicit solution derived in the previous section. While it will become clear that for this case the approach of Section 3.1 leads to the result with considerably less effort, a comparison of the two approaches gives rise to identities between special functions which are interesting in their own right.

From $\widetilde{f_{Y}}(s)=\alpha /(s+\alpha)$ one gets after standard algebraic manipulations

$\widetilde{V}(s)=e^{-\frac{(c-\beta)}{q} s} s^{\frac{\delta}{q}-1}(s+\alpha)^{\frac{\lambda}{q}}\left(C+\int\left(\frac{(c-\beta) V(0)-\frac{q}{s^{2}}-\frac{\beta}{s}}{q}\right) e^{\frac{(c-\beta)}{q} s} s^{-\frac{\delta}{q}}(s+\alpha)^{-\frac{\lambda}{q}} d s\right)$.

Expanding the exponential term inside the integral gives

$$
\begin{gathered}
\widetilde{V}(s)=e^{-\frac{(c-\beta)}{q} s} s^{\frac{\delta}{q}-1}(s+\alpha)^{\frac{\lambda}{q}}\left(C+\frac{(c-\beta)}{q} V(0) \sum_{n=0}^{\infty} \frac{\left(\frac{c-\beta}{q}\right)^{n}}{n !} \int s^{n-\frac{\delta}{q}}(s+\alpha)^{-\frac{\lambda}{q}} d s\right. \\
\left.-\sum_{n=0}^{\infty} \frac{\left(\frac{c-\beta}{q}\right)^{n}}{n !} \int s^{n-\frac{\delta}{q}-2}(s+\alpha)^{-\frac{\lambda}{q}} d s-\frac{\beta}{q} \sum_{n=0}^{\infty} \frac{\left(\frac{c-\beta}{q}\right)^{n}}{n !} \int s^{n-\frac{\delta}{q}-1}(s+\alpha)^{-\frac{\lambda}{q}} d s\right) .
\end{gathered}
$$

The following lemma establishes a connection between the integrals in (18) and the Gauss hypergeometric function ${ }_{2} F_{1}$. Recall (also for later use) that the generalized hypergeometric function ${ }_{p} F_{q}$ is defined through

$$
{ }_{p} F_{q}\left(a_{1}, \ldots, a_{p} ; b_{1}, \ldots, b_{q} ; z\right)=\sum_{n=0}^{\infty} \frac{\left(a_{1}\right)_{n} \cdots\left(a_{p}\right)_{n}}{\left(b_{1}\right)_{n} \cdots\left(b_{q}\right)_{n}} \frac{z^{n}}{n !} .
$$

Lemma 4.1. For $(n, k) \in \mathbb{N}_{0} \times \mathbb{N}_{0}$ and $n-\frac{\delta}{q}-k \notin \mathbb{Z}^{-}$, one has

$$
\begin{aligned}
& \int s^{n-\frac{\delta}{q}-k}(s+\alpha)^{-\frac{\lambda}{q}} d s=\frac{\alpha^{-\frac{\lambda}{q}}}{\left(n-\frac{\delta}{q}-k+1\right)} s^{n-\frac{\delta}{q}-k+1}{ }_{2} F_{1}\left(\frac{\lambda}{q} ; n-\frac{\delta}{q}-k+1, n-\frac{\delta}{q}-k+2 ;-\frac{s}{\alpha}\right) \\
& \text { for } s \in \mathfrak{S}=\left\{s:\left|\frac{s}{\alpha}\right|<1, s \neq 0\right\}
\end{aligned}
$$


Proof. Let $(n, k) \in \mathbb{N}_{0} \times \mathbb{N}_{0}$ and define the new variable $\xi=s / \alpha$, i.e.

$$
\begin{aligned}
& \int s^{n-\frac{\delta}{q}-k}(s+\alpha)^{-\frac{\lambda}{q}} d s=\alpha^{n-\frac{\delta+\lambda}{q}-k+1} \int \xi^{n-\frac{\delta}{q}-k}(1+\xi)^{-\frac{\lambda}{q}} d \xi \\
&=\alpha^{-\frac{\lambda}{q}}(\alpha \xi)^{n-\frac{\delta}{q}-k+1} \sum_{j=0}^{\infty} \frac{\left(\frac{\lambda}{q}\right)_{j}}{\left(n-\frac{\delta}{q}-k+1+j\right)} \frac{(-\xi)^{j}}{j !}
\end{aligned}
$$

In terms of the original variable $s$, this can be recast into the form

$$
\frac{\alpha^{-\frac{\lambda}{q}}}{\left(n-\frac{\delta}{q}-k+1\right)} s^{n-\frac{\delta}{q}-k+1} \sum_{j=0}^{\infty} \frac{\left(\frac{\lambda}{q}\right)_{j}\left(n-\frac{\delta}{q}-k+1\right)_{j}}{\left(n-\frac{\delta}{q}-k+2\right)_{j}} \frac{\left(-\frac{s}{\alpha}\right)^{j}}{j !} .
$$

Using Lemma 4.1, we can rewrite (18) as

$$
\begin{aligned}
\widetilde{V}(s) & =e^{-\frac{(c-\beta)}{q} s}\left(C s^{\frac{\delta}{q}-1}(s+\alpha)^{\frac{\lambda}{q}}\right. \\
& +\frac{(c-\beta)}{q} V(0)\left(1+\frac{s}{\alpha}\right)^{\frac{\lambda}{q}} \sum_{n=0}^{\infty} \frac{\left(\frac{c-\beta}{q}\right)^{n}}{n !} \frac{s^{n}}{\left(n-\frac{\delta}{q}+1\right)}{ }_{2} F_{1}\left(\frac{\lambda}{q}, n-\frac{\delta}{q}+1 ; n-\frac{\delta}{q}+2 ;-\frac{s}{\alpha}\right) \\
& -\left(1+\frac{s}{\alpha}\right)^{\frac{\lambda}{q}} \frac{1}{s^{2}} \sum_{n=0}^{\infty} \frac{\left(\frac{c-\beta}{q}\right)^{n}}{n !} \frac{s^{n}}{\left(n-\frac{\delta}{q}-1\right)}{ }_{2} F_{1}\left(\frac{\lambda}{q}, n-\frac{\delta}{q}-1 ; n-\frac{\delta}{q} ;-\frac{s}{\alpha}\right) \\
& \left.-\left(1+\frac{s}{\alpha}\right)^{\frac{\lambda}{q}} \frac{\beta}{q s} \sum_{n=0}^{\infty} \frac{\left(\frac{c-\beta}{q}\right)^{n}}{n !} \frac{s^{n}}{\left(n-\frac{\delta}{q}\right)}{ }_{2} F_{1}\left(\frac{\lambda}{q}, n-\frac{\delta}{q} ; n-\frac{\delta}{q}+1 ;-\frac{s}{\alpha}\right)\right) .
\end{aligned}
$$

Denote

$$
{ }_{2} \mathbf{F}_{\mathbf{1}}(a, b ; \nu ; z):=\frac{{ }_{2} F_{1}(\nu-a, \nu-b ; \nu ; z)}{\Gamma(\nu)}
$$

Successively using the transformation formulas

$$
{ }_{2} \mathbf{F}_{\mathbf{1}}(a, b ; \nu ; z)=(1-z)^{\nu-a-b}{ }_{2} \mathbf{F}_{\mathbf{1}}(\nu-a, \nu-b ; \nu ; z)
$$


and

$$
\begin{aligned}
{ }_{2} \mathbf{F}_{\mathbf{1}}(a, b ; \nu ; z) & =\frac{\pi}{\sin (\pi(b-a))}\left[\frac{(-z)^{-a}}{\Gamma(b) \Gamma(\nu-a)}{ }_{2} \mathbf{F}_{\mathbf{1}}\left(a, a-\nu+1 ; a-b+1 ; \frac{1}{z}\right)\right. \\
& \left.-\frac{(-z)^{-a}}{\Gamma(b) \Gamma(\nu-a)}{ }_{2} \mathbf{F}_{\mathbf{1}}\left(a, a-\nu+1 ; a-b+1 ; \frac{1}{z}\right)\right],
\end{aligned}
$$

(cf. [21]), we can then rewrite (19) as

$$
\begin{aligned}
\widetilde{V}(s) & =e^{\frac{(c-\beta)}{q} s}\left[\left(C+\frac{(c-\beta)}{q} V(0) \alpha^{-\frac{\lambda+\delta}{q}+1} \sum_{n=0}^{\infty} \frac{(z(0))^{n}}{n !} \frac{\Gamma\left(-(n+1)+\frac{\lambda+\delta}{q}\right) \Gamma\left(n-\frac{\delta}{q}+1\right)}{\Gamma\left(\frac{\lambda}{q}\right)}\right.\right. \\
& -\alpha^{-\frac{\lambda+\delta}{q}-1} \sum_{n=0}^{\infty} \frac{(z(0))^{n}}{n !} \frac{\Gamma\left(-n+1+\frac{\lambda+\delta}{q}\right) \Gamma\left(n-\frac{\delta}{q}-1\right)}{\Gamma\left(\frac{\lambda}{q}\right)} \\
& \left.-\frac{\beta}{q} \alpha^{-\frac{\lambda+\delta}{q}} \sum_{n=0}^{\infty} \frac{(z(0))^{n}}{n !} \frac{\Gamma\left(-n+\frac{\lambda+\delta}{q}\right) \Gamma\left(n-\frac{\delta}{q}\right)}{\Gamma\left(\frac{\lambda}{q}\right)}\right) s^{\frac{\delta}{q}-1}(s+\alpha)^{\frac{\lambda}{q}} \\
& -\frac{(c-\beta)}{q} V(0)\left(1+\frac{\alpha}{s}\right) \sum_{n=0}^{\infty} \frac{\left(\frac{(c-\beta)}{q}\right)^{n}}{n !} \frac{s^{n}}{\left(-(n+1)+\frac{\lambda+\delta}{q}\right)}{ }_{2} F_{1}\left(1, \frac{\delta}{q}-n ; \frac{\lambda+\delta}{q}-n ;-\frac{\alpha}{s}\right) \\
& +\frac{\left(1+\frac{\alpha}{s}\right)}{s^{2}} \sum_{n=0}^{\infty} \frac{\left(\frac{(c-\beta)}{q}\right)^{n}}{n !} \frac{s^{n}}{\left(-n+1+\frac{\lambda+\delta}{q}\right)}{ }_{2} F_{1}\left(1, \frac{\delta}{q}-n+2 ; \frac{\lambda+\delta}{q}-n+2 ;-\frac{\alpha}{s}\right) \\
& \left.+\frac{\beta}{q} \frac{\left(1+\frac{\alpha}{s}\right)}{s} \sum_{n=0}^{\infty} \frac{\left(\frac{(c-\beta)}{q}\right)^{n}}{n !} \frac{s^{n}}{\left(-n+\frac{\lambda+\delta}{q}\right)}{ }_{2} F_{1}\left(1, \frac{\delta}{q}-n+1 ; \frac{\lambda+\delta}{q}-n+1 ;-\frac{\alpha}{s}\right)\right] .
\end{aligned}
$$

Since

$$
\begin{aligned}
\sum_{n=0}^{\infty} \frac{z^{n}}{n !} \frac{\Gamma\left(-n+\kappa+\frac{\lambda+\delta}{q}\right) \Gamma\left(n-\frac{\delta}{q}-\kappa\right)}{\Gamma\left(\frac{\lambda}{q}\right)} & \\
& =\frac{\Gamma\left(-\frac{\delta}{q}-\kappa\right) \Gamma\left(\kappa+\frac{\lambda+\delta}{q}\right)}{\Gamma\left(\frac{\lambda}{q}\right)} M\left(-\frac{\delta}{q}-\kappa, 1-\kappa-\frac{\lambda+\delta}{q},-z\right),
\end{aligned}
$$


equation (20) simplifies to

$$
\begin{aligned}
\widetilde{V}(s) & =e^{-\frac{(c-\beta)}{q} s}\left[s^{\frac{\delta}{q}-1}(s+\alpha)^{\frac{\lambda}{q}}(C+D)\right. \\
& -\frac{(c-\beta)}{q} V(0)\left(1+\frac{\alpha}{s}\right) \sum_{n=0}^{\infty} \frac{\left(\frac{(c-\beta)}{q}\right)^{n}}{n !} \frac{s^{n}}{\left(-(n+1)+\frac{\lambda+\delta}{q}\right)}{ }_{2} F_{1}\left(1, \frac{\delta}{q}-n ; \frac{\lambda+\delta}{q}-n ;-\frac{\alpha}{s}\right) \\
& +\frac{\left(1+\frac{\alpha}{s}\right)}{s^{2}} \sum_{n=0}^{\infty} \frac{\left(\frac{(c-\beta)}{q}\right)^{n}}{n !} \frac{s^{n}}{\left(-n+1+\frac{\lambda+\delta}{q}\right)}{ }_{2} F_{1}\left(1, \frac{\delta}{q}-n+2 ; \frac{\lambda+\delta}{q}-n+2 ;-\frac{\alpha}{s}\right) \\
& \left.+\frac{\beta\left(1+\frac{\alpha}{s}\right)}{s} \sum_{n=0}^{\infty} \frac{\left(\frac{(c-\beta)}{q}\right)^{n}}{n !} \frac{s^{n}}{\left(-n+\frac{\lambda+\delta}{q}\right)}{ }_{2} F_{1}\left(1, \frac{\delta}{q}-n+1 ; \frac{\lambda+\delta}{q}-n+1 ;-\frac{\alpha}{s}\right)\right]
\end{aligned}
$$

where

$$
\begin{gathered}
D=\frac{(c-\beta)}{q} V(0) \alpha^{-\frac{\lambda+\delta}{q}+1} \frac{\Gamma\left(1-\frac{\delta}{q}\right) \Gamma\left(\frac{\lambda+\delta}{q}-1\right)}{\Gamma\left(\frac{\lambda}{q}\right)} M\left(1-\frac{\delta}{q}, 2-\frac{\lambda+\delta}{q},-\frac{\alpha(c-\beta)}{q}\right) \\
-\alpha^{-\frac{\lambda+\delta}{q}-1} \frac{\Gamma\left(-1-\frac{\delta}{q}\right) \Gamma\left(\frac{\lambda+\delta}{q}+1\right)}{\Gamma\left(\frac{\lambda}{q}\right)} M\left(-1-\frac{\delta}{q},-\frac{\lambda+\delta}{q},-\frac{\alpha(c-\beta)}{q}\right) \\
-\frac{\beta}{q} \alpha^{-\frac{\lambda+\delta}{q}} \frac{\Gamma\left(-\frac{\delta}{q}\right) \Gamma\left(\frac{\lambda+\delta}{q}\right)}{\Gamma\left(\frac{\lambda}{q}\right)} M\left(-\frac{\delta}{q}, 1-\frac{\lambda+\delta}{q},-\frac{\alpha(c-\beta)}{q}\right) .
\end{gathered}
$$

From

$$
\frac{1}{\Gamma(b)} \int_{0}^{\infty} e^{-s x} x^{b-1} M(a, b, x) d x=s^{a-b}(s-1)^{-a}, \quad \Re(b)>0, \Re(s)>1,
$$


one deduces that for $\lambda+\delta<q$ and $\Re(s)>0$, the inverse Laplace transform of $s^{\frac{\delta}{q}-1}(s+\alpha)^{\frac{\lambda}{q}}$ is given by

$$
\begin{aligned}
\Re\left\{\left(\frac{\Gamma\left(\frac{\delta}{q}\right)}{\Gamma\left(\frac{\lambda+\delta}{q}\right)} U\left(\frac{\delta}{q}, 1+\frac{\lambda+\delta}{q},-\alpha x\right)\right.\right. \\
\\
\left.\left.\quad-\frac{\Gamma\left(\frac{\delta}{q}\right)}{\Gamma\left(\frac{\lambda+\delta}{q}\right)} \frac{\Gamma\left(-\frac{\lambda+\delta}{q}\right)}{\Gamma\left(-\frac{\lambda}{q}\right)} M\left(\frac{\delta}{q}, 1+\frac{\lambda+\delta}{q},-\alpha x\right)\right) \frac{(-\alpha)^{\frac{\lambda+\delta}{q}}}{\Gamma\left(1-\frac{\lambda+\delta}{q}\right)}\right\} .
\end{aligned}
$$

For the sake of brevity, define $g(x):=x^{-\frac{\lambda+\delta}{q}} M\left(-\frac{\lambda}{q}, 1-\frac{\lambda+\delta}{q},-\alpha x\right) / \Gamma\left(1-\frac{\lambda+\delta}{q}\right)$. The first term of (21) can be interpreted as

$$
e^{-\frac{(c-\beta)}{q} s} \tilde{g}(s)=\mathcal{L}\left\{u\left(x-\frac{c-\beta}{q}\right) g\left(x-\frac{c-\beta}{q}\right)\right\}
$$

where $u$ is the Heaviside function. Since $u\left(x-\frac{c-\beta}{q}\right) g\left(x-\frac{c-\beta}{q}\right)$ becomes unbounded as $x$ approaches $(c-\beta) / q$ from the right, the linear boundedness of $V(x)$ imposes $C=-D$.

The three hypergeometric functions ${ }_{2} F_{1}$ in (21) have parameters differing by an integer. A connection between those terms is given by the identity

$$
\begin{aligned}
{ }_{2} F_{1}\left(1, \frac{\delta}{q}-n ; \frac{\lambda+\delta}{q}-n ;-\frac{\alpha}{s}\right) & =\sum_{k=0}^{m-1} \frac{\left(n+1-k-\frac{\delta}{q}\right)_{k}}{\left(n+1-k-\frac{\lambda+\delta}{q}\right)_{k}}\left(-\frac{\alpha}{s}\right)^{k} \\
& +\left(-\frac{\alpha}{s}\right)^{m} \frac{\left(1-\frac{\delta}{q}+n-m\right)_{m}}{\left(1-\frac{\lambda+\delta}{q}+n-m\right)_{m} F_{1}\left(1, \frac{\delta}{q}-n+m ; \frac{\lambda+\delta}{q}-n+m ;-\frac{\alpha}{s}\right)}
\end{aligned}
$$


for $(n, m) \in \mathbb{N}_{0} \times \mathbb{N}$. Respective substitution in (21) gives

$$
\begin{aligned}
\widetilde{V}(s) & =e^{-\frac{(c-\beta)}{q} s}\left[-\frac{(c-\beta)}{q} V(0)\left(1+\frac{\alpha}{s}\right) \sum_{n=0}^{\infty} \frac{\left(\frac{(c-\beta)}{q}\right)^{n}}{n !} \frac{s^{n}}{\left(-(n+1)+\frac{\lambda+\delta}{q}\right)} .\right. \\
& +\frac{\left(1+\frac{\alpha}{s}\right)}{\alpha^{2}} \sum_{n=0}^{\infty} \frac{\left(\frac{(c-\beta)}{q}\right)^{n}}{n !} \frac{s^{n}\left(1-{ }_{2} F_{1}\left(1, \frac{\delta}{q}-n ; \frac{\lambda+\delta}{q}-n ;-\frac{\alpha}{s}\right)\right.}{\left(n-\frac{\delta}{q}-1\right)\left(n-\frac{\delta}{q}\right)} \\
& -\frac{\left(1+\frac{\alpha}{s}\right)}{\alpha s} \sum_{n=0}^{\infty} \frac{\left(\frac{(c-\beta)}{q}\right)^{n}}{n !} \frac{s^{n}}{\left(n-\frac{\delta}{q}-1\right)} \\
& \left.-\frac{\beta}{q} \frac{\left(1+\frac{\alpha}{s}\right)}{\alpha} \sum_{n=0}^{\infty} \frac{\left(\frac{(c-\beta)}{q}\right)^{n}}{n !} \frac{s^{n}\left(1-{ }_{2} F_{1}\left(1, \frac{\delta}{q}-n ; \frac{\lambda+\delta}{q}-n ;-\frac{\alpha}{s}\right)\right)}{\left(n-\frac{\delta}{q}\right)}\right] .
\end{aligned}
$$

With considerable effort, the latter expression can be represented as

$$
\begin{aligned}
\widetilde{V}(s) & =e^{-\frac{(c-\beta)}{q} s}\left(1+\frac{\alpha}{s}\right)\left[B \cdot{ }_{2} F_{1}\left(1, \frac{\delta}{q} ; \frac{\lambda+\delta}{q} ;-\frac{\alpha}{s}\right)\right. \\
& +\frac{(c-\beta)}{q} V(0) \sum_{n=1}^{\infty} \frac{\left(\frac{(c-\beta)}{q}\right)^{n} s^{n}}{n !\left(n+1-\frac{\lambda+\delta}{q}\right)}{ }_{2} F_{2}\left(1, n+1-\frac{\delta}{q} ; n+1, n+2-\frac{\lambda+\delta}{q} ;-\frac{\alpha(c-\beta)}{q}\right) \\
& -\left(\frac{c-\beta}{q}\right)^{2} \sum_{n=1}^{\infty} \frac{\left(\frac{(c-\beta)}{q}\right)^{n} s^{n}}{(n+2) !\left(n+1-\frac{\lambda+\delta}{q}\right)}{ }_{2} F_{2}\left(1, n+1-\frac{\delta}{q} ; n+3, n+2-\frac{\lambda+\delta}{q} ;-\frac{\alpha(c-\beta)}{q}\right) \\
& -\frac{\beta}{q} \frac{(c-\beta)}{q} \sum_{n=1}^{\infty} \frac{\left(\frac{(c-\beta)}{q}\right)^{n} s^{n}}{(n+1) !\left(n+1-\frac{\lambda+\delta}{q}\right)}{ }_{2} F_{2}\left(1, n+1-\frac{\delta}{q} ; n+2, n+2-\frac{\lambda+\delta}{q} ;-\frac{\alpha(c-\beta)}{q}\right) \\
& \left.+\frac{\alpha s\left(1+\frac{\delta}{q}\right)}{\alpha}-\frac{q(\lambda+\delta)}{\alpha^{2} \delta(q+\delta)}+\frac{c}{\alpha \delta}\right],
\end{aligned}
$$


where

$$
\begin{aligned}
B & =\frac{(c-\beta)}{q} V(0) \frac{M\left(1-\frac{\delta}{q}, 2-\frac{\lambda+\delta}{q},-\frac{\alpha(c-\beta)}{q}\right)}{\left(1-\frac{\lambda+\delta}{q}\right)}+\frac{q(\lambda+\delta)}{\alpha^{2} \delta(q+\delta)} M\left(-1-\frac{\delta}{q},-\frac{\lambda+\delta}{q},-\frac{\alpha(c-\beta)}{q}\right) \\
& -\frac{\beta}{\alpha \delta} M\left(-\frac{\delta}{q}, 1-\frac{\lambda+\delta}{q},-\frac{\alpha(c-\beta)}{q}\right) .
\end{aligned}
$$

Using the contiguous relation

$$
\nu(1-z){ }_{2} F_{1}(a, b ; \nu ; z)=\nu{ }_{2} F_{1}(a-1, b ; \nu ; z)-(b-\nu) z_{2} F_{1}(a, b ; \nu+1 ; z)
$$

for our context, we obtain

$$
{ }_{2} F_{1}\left(1, \frac{\delta}{q} ; \frac{\lambda+\delta}{q} ;-\frac{\alpha}{s}\right)=\frac{1}{1+\frac{\alpha}{s}}\left(1+\frac{\alpha \lambda}{\lambda+\delta} \frac{{ }_{2} F_{1}\left(1, \frac{\delta}{q} ; 1+\frac{\lambda+\delta}{q} ;-\frac{\alpha}{s}\right)}{s}\right),
$$

which transforms (22) into

$$
\begin{aligned}
\widetilde{V}(s) & =e^{-\frac{(c-\beta)}{q} s} \frac{B \alpha \lambda}{(\lambda+\delta)} \frac{{ }_{2} F_{1}\left(1, \frac{\delta}{q} ; 1+\frac{\lambda+\delta}{q} ;-\frac{\alpha}{s}\right)}{s} \\
& +e^{-\frac{(c-\beta)}{q} s}\left\{B+\left(1+\frac{\alpha}{s}\right)[\right. \\
& \frac{(c-\beta)}{q} V(0) \sum_{n=1}^{\infty} \frac{\left(\frac{(c-\beta)}{q}\right)^{n} s^{n}}{n !\left(n+1-\frac{\lambda+\delta}{q}\right)}{ }_{2} F_{2}\left(1, n+1-\frac{\delta}{q} ; n+1, n+2-\frac{\lambda+\delta}{q} ;-\frac{\alpha(c-\beta)}{q}\right) \\
& -\left(\frac{c-\beta}{q}\right)^{2} \sum_{n=1}^{\infty} \frac{\left(\frac{(c-\beta)}{q}\right)^{n} s^{n}}{(n+2) !\left(n+1-\frac{\lambda+\delta}{q}\right)}{ }_{2} F_{2}\left(1, n+1-\frac{\delta}{q} ; n+3, n+2-\frac{\lambda+\delta}{q} ;-\frac{\alpha(c-\beta)}{q}\right) \\
& -\frac{\beta}{q} \frac{(c-\beta)}{q} \sum_{n=1}^{\infty} \frac{\left(\frac{(c-\beta)}{q}\right)^{n} s^{n}}{(n+1) !\left(n+1-\frac{\lambda+\delta}{q}\right)}{ }_{2} F_{2}\left(1, n+1-\frac{\delta}{q} ; n+2, n+2-\frac{\lambda+\delta}{q} ;-\frac{\alpha(c-\beta)}{q}\right) \\
& \left.\left.+\frac{1}{\alpha s\left(1+\frac{\delta}{q}\right)}-\frac{q(\lambda+\delta)}{\alpha^{2} \delta(q+\delta)}+\frac{c}{\alpha \delta}\right\}\right)
\end{aligned}
$$

Utilizing the relationship (cf. Olver [21])

$$
\int_{0}^{\infty} e^{-s x} x^{b-1} \mathbf{M}(a, \nu, k x) d x=\frac{\Gamma(b)}{s^{b}}{ }_{2} \mathbf{F}_{\mathbf{1}}\left(a, b ; \nu ; \frac{k}{s}\right), \quad \Re(b)>0, \Re(s)>\max (\Re(k), 0),
$$


gives the inverse Laplace transform of the first term of (23):

$$
\begin{aligned}
& e^{-\frac{(c-\beta)}{q} s} \frac{B \alpha \lambda}{(\lambda+\delta)} \frac{{ }_{2} F_{1}\left(1, \frac{\delta}{q} ; 1+\frac{\lambda+\delta}{q} ;-\frac{\alpha}{s}\right)}{s} \\
& \quad=\frac{B \alpha \lambda}{(\lambda+\delta)} \mathcal{L}\left\{u\left(x-\frac{c-\beta}{q}\right) M\left(\frac{\delta}{q}, 1+\frac{\lambda+\delta}{q},-\alpha\left(x-\frac{c-\beta}{q}\right)\right)\right\} .
\end{aligned}
$$

Since we know by different means from Section 3 the expression for $V(x)$, we can take the (direct) Laplace transform of (17) in order to compare it with the above expression. After some efforts, one obtains from (17)

$\tilde{V}(s)=e^{-\frac{(c-\beta)}{q} s} \tilde{C}\left[H(s)+\frac{{ }_{2} F_{1}\left(\frac{\delta}{q}, 1 ; 1+\frac{\lambda+\delta}{q} ;-\frac{\alpha}{s}\right)}{s}\right]+\frac{1}{q+\delta}\left(\frac{q}{s^{2}}+\frac{\beta}{s}+\frac{q\left(c-\frac{\lambda}{\alpha}\right)}{\delta s}\right)$,

where

$$
H(s):=\int_{-\frac{(c-\beta)}{q}}^{0} e^{-s y} M\left(\frac{\delta}{q}, 1+\frac{\lambda+\delta}{q},-\alpha y\right) d y
$$

One can show that $\frac{B \alpha \lambda}{(\lambda+\delta)}=\tilde{C}$ and

$$
H(s)=\sum_{n=0}^{\infty} \frac{\left(\frac{c-\beta}{q}\right)^{n+1} s^{n}}{(n+1) !}{ }_{2} F_{2}\left(n+1, \frac{\delta}{q} ; 2+n, 1+\frac{\lambda+\delta}{q} ; \frac{\alpha(c-\beta)}{q}\right)
$$


so the first term coincides with the one in (23). Since Expressions (24) and (23) have to coincide altogether, this leads to the identity

$$
\begin{aligned}
& e^{-\frac{(c-\beta)}{q} s} \frac{B \alpha \lambda}{(\lambda+\delta)} H(s)+\frac{1}{q+\delta}\left(\frac{q}{s^{2}}+\frac{\beta}{s}+\frac{q\left(c-\frac{\lambda}{\alpha}\right)}{\delta s}\right)=e^{-\frac{(c-\beta)}{q} s}\left\{B+\left(1+\frac{\alpha}{s}\right)[\right. \\
& \quad \frac{(c-\beta)}{q} V(0) \sum_{n=1}^{\infty} \frac{\left(\frac{(c-\beta)}{q}\right)^{n} s^{n}}{n !\left(n+1-\frac{\lambda+\delta}{q}\right)}{ }_{2} F_{2}\left(1, n+1-\frac{\delta}{q} ; n+1, n+2-\frac{\lambda+\delta}{q} ;-\frac{\alpha(c-\beta)}{q}\right) \\
& -\left(\frac{c-\beta}{q}\right)^{2} \sum_{n=1}^{\infty} \frac{\left(\frac{(c-\beta)}{q}\right)^{n} s^{n}}{(n+2) !\left(n+1-\frac{\lambda+\delta}{q}\right)}{ }_{2} F_{2}\left(1, n+1-\frac{\delta}{q} ; n+3, n+2-\frac{\lambda+\delta}{q} ;-\frac{\alpha(c-\beta)}{q}\right) \\
& -\frac{\beta}{q} \frac{(c-\beta)}{q} \sum_{n=1}^{\infty} \frac{\left(\frac{(c-\beta)}{q}\right)^{n} s^{n}}{(n+1) !\left(n+1-\frac{\lambda+\delta}{q}\right)}{ }_{2} F_{2}\left(1, n+1-\frac{\delta}{q} ; n+2, n+2-\frac{\lambda+\delta}{q} ;-\frac{\alpha(c-\beta)}{q}\right) \\
& \left.\left.+\frac{1}{\alpha s\left(1+\frac{\delta}{q}\right)}-\frac{q(\lambda+\delta)}{\alpha^{2} \delta(q+\delta)}+\frac{c}{\alpha \delta}\right]\right\} .
\end{aligned}
$$

While it is far from obvious to show analytically that (25) holds true, it is indeed the case, as numerical verifications show. In fact, the two alternative approaches of Sections 3 and 4 - through identity (25) - suggest new relations between ${ }_{2} F_{2^{-}}$ hypergeometric functions of argument $\pm z$. A detailed study of such relations is, however, beyond the scope of this paper.

\section{The TIME OF RUIN}

Let us now study the effect of the proposed dividend strategy on the distribution of the ruin time $\tau_{x}$. For this purpose, consider the expected discounted penalty at ruin

$$
m_{\delta}(x):=\mathbb{E}\left[e^{-\delta \tau_{x}} w\left(\left|X_{\tau_{x}}\right|\right)\right],
$$

where $w$ is a non-negative penalty function of the deficit at ruin. Given differentiability of $m_{\delta}(x)$, the standard arguments based on the infinitesimal generator then lead to the integro-differential equation

$$
(c-(q x+\beta)) m_{\delta}^{\prime}(x)-(\lambda+\delta) m_{\delta}(x)+\lambda \int_{0}^{x} m_{\delta}(x-y) d F_{Y}(y)=-\lambda A(x), \quad x \geq 0,
$$


where

$$
A(x):=\int_{x}^{\infty} w(y-x) d F_{Y}(y) .
$$

We will again restrict our considerations to exponentially distributed claims with parameter $\alpha>0$. In this case, $\left|X_{\tau_{x}}\right| \sim \operatorname{Exp}(\alpha)$ due to lack of memory, so that we can focus on the (Laplace transform of the) time of ruin, i.e. $w(x)=1$.

Similarly to Section 3.1, this leads to the second-order homogeneous differential equation

$(c-(q x+\beta)) m_{\delta}^{\prime \prime}(x)+[\alpha(c-(q x+\beta))-(q+\lambda+\delta)] m_{\delta}^{\prime}(x)-\alpha \delta m_{\delta}(x)=0, \quad x \geq 0$.

Because of the linear boundedness of $m_{\delta}(x)$ in $x$, the solution to (27) matches the homogeneous solution $V_{h}(x):=V(x)-V_{p}(x)$ to (11) up to a constant factor. That is, for $x \geq 0$, we can write $m_{\delta}(x)=B V_{h}(x)$ for some constant $B$. Letting $x=0$ in (26) yields

$$
(c-\beta) m_{\delta}^{\prime}(0)-(\lambda+\delta) m_{\delta}(0)=-\lambda,
$$

that is

$$
(c-\beta) B\left(V^{\prime}(0)-V_{p}^{\prime}(0)\right)-(\lambda+\delta) B\left(V(0)-V_{p}(0)\right)=-\lambda
$$

and hence

$$
B=\frac{\lambda}{\beta+\frac{q(c-\beta)}{q+\delta}-\frac{\lambda+\delta}{q+\delta}\left(\beta+\frac{q}{\delta}\left(c-\frac{\lambda}{\alpha}\right)\right)} .
$$

Proposition 5.1. For any $x \geq 0$, the Laplace transform of the ruin time in a Cramér-Lundberg model with affine dividend strategy (2) and Exp( $\alpha)$-distributed claims is given by

$$
m_{\delta}(x)=\frac{\lambda M\left(\frac{\delta}{q}, 1+\frac{\lambda+\delta}{q}, z(x)\right)}{\frac{\alpha \delta(c-\beta)}{q+\lambda+\delta} M\left(1+\frac{\delta}{q}, 2+\frac{\lambda+\delta}{q}, z(0)\right)+(\lambda+\delta) M\left(\frac{\delta}{q}, 1+\frac{\lambda+\delta}{q}, z(0)\right)}, \quad x \geq 0,
$$

where $z(x)=\frac{\alpha(c-(q x+\beta))}{q}$.

One particular quantity of interest is the expected ruin time. While it can be simply obtained by taking the derivative

$$
\mathbb{E}\left[\tau_{x}\right]=-\left.\frac{d}{d \delta} m_{\delta}(x)\right|_{\delta=0}
$$


the concrete calculation is considerably involved. It requires differentiating $M$ with respect to its first two parameters:

$$
M^{(a)}=\frac{d}{d a} M(a, b, z), \quad M^{(b)}=\frac{d}{d b} M(a, b, z) .
$$

The so-called Kummer transformation $M(a, b, z)=e^{z} M(b-a, b,-z)$ will facilitate the mathematical tractability, under which $(28)$ reads

$$
m_{\delta}(x)=\frac{\lambda e^{-\alpha x} M\left(a, a+\frac{\delta}{q},-z(x)\right)}{\frac{\alpha \delta(c-\beta)}{q+\lambda+\delta} M\left(a, a+1+\frac{\delta}{q},-z(0)\right)+(\lambda+\delta) M\left(a, a+\frac{\delta}{q},-z(0)\right)}, \quad x \geq 0,
$$

where $a=1+\frac{\lambda}{q}$.

We now discuss two possible representations for the derivative of $M$ :

5.1. Digamma functions. A first and customary approach to calculate $M^{(a)}$ makes use of the derivative of the Pochhammer symbol $a_{(n)}:=\Gamma(a+n) / \Gamma(n)$, which is given by

$$
\frac{d(a)_{n}}{d a}=(a)_{n}[\psi(a+n)-\psi(a)]
$$

where $\psi(a)=\frac{d}{d a} \log \Gamma(a)=\frac{\Gamma^{\prime}(a)}{\Gamma(a)}$ is the logarithmic derivative of the gamma function, known as the digamma function. Next, using the power series definition of $M$ given in (14) leads to the representations

$$
M^{(a)}=\sum_{n=0}^{\infty}[\psi(a+n)-\psi(a)] \frac{(a)_{n}}{(b)_{n}} \frac{z^{n}}{n !},
$$

and

$$
M^{(b)}=\sum_{n=0}^{\infty}[\psi(b)-\psi(b+n)] \frac{(a)_{n}}{(b)_{n}} \frac{z^{n}}{n !} .
$$

With that in mind, the derivative of (29) with respect to $\delta$ (together with the property $\left.M(a, a, z)=e^{z}\right)$ yields

$$
\begin{aligned}
\left.\frac{d}{d \delta} m_{\delta}(x)\right|_{\delta=0} & =\frac{e^{z(x)}}{q} \sum_{n=0}^{\infty}[\psi(a)-\psi(a+n)] \frac{(-z(x))^{n}}{n !}-\frac{\alpha(c-\beta)}{\lambda(q+\lambda)} e^{z(0)} M(a, a+1,-z(0)) \\
& -\frac{1}{\lambda}-\frac{e^{z(0)}}{q} \sum_{n=0}^{\infty}[\psi(a)-\psi(a+n)] \frac{(-z(0))^{n}}{n !}
\end{aligned}
$$


But for series of the above type there are expressions in terms of the generalized hypergeometric function ${ }_{2} F_{2}$ available (cf. [10]):

$$
\sum_{n=0}^{\infty}[\psi(a+n)-\psi(a)] \frac{(z(x))^{n}}{n !}=\frac{z(x)}{a} e^{z(x)}{ }_{2} F_{2}(1,1 ; 2, a+1 ;-z(x)) .
$$

Using this last result together with the relation $M(a, a+1,-z)=a z^{-a} \gamma(a, z)$ (where $\gamma(a, z)$ denotes the lower incomplete gamma function) leads to the following formula:

Proposition 5.2. For any $x \geq 0$, the expected time of ruin under the the proposed dividend strategy in model (1) with exponentially distributed claims with parameter $\alpha$ is given by

$$
\begin{aligned}
\mathbb{E}\left[\tau_{x}\right]=\frac{1+e^{z(0)} \gamma\left(1+\frac{\lambda}{q}, z(0)\right) z(0)^{-\frac{\lambda}{q}}}{\lambda} & \\
& +\frac{{ }_{2} F_{2}\left(1,1 ; 2,2+\frac{\lambda}{q} ; z(0)\right) z(0)-{ }_{2} F_{2}\left(1,1 ; 2,2+\frac{\lambda}{q} ; z(x)\right) z(x)}{q+\lambda}
\end{aligned}
$$

where $z(x)=\frac{\alpha(c-(q x+\beta)}{q}$.

5.2. Kampé de Fériet functions. As an alternative, one can express the derivatives of the Kummer function also in terms of the bivariate Kampé de Fériet function

$$
\begin{array}{r}
F_{R, S, U}^{A, B, D}\left(\begin{array}{l}
\left.a_{1}, \ldots, a_{A} ; b_{1}, \ldots, b_{B} ; d_{1}, \ldots, d_{D} ; x, y\right) \\
r_{1}, \ldots, r_{R} ; s_{1}, \ldots, s_{S} ; u_{1}, \ldots, u_{U} ;
\end{array}\right) \\
\quad=\sum_{m=0}^{\infty} \sum_{n=0}^{\infty} \frac{\prod_{j=1}^{A}\left(a_{j}\right)_{m+n} \prod_{j=1}^{B}\left(b_{j}\right)_{m} \prod_{j=1}^{D}\left(d_{j}\right)_{n}}{\prod_{j=1}^{R}\left(r_{j}\right)_{m+n} \prod_{j=1}^{S}\left(s_{j}\right)_{m} \prod_{j=1}^{U}\left(u_{j}\right)_{n}} \frac{x^{m} y^{n}}{m ! n !},
\end{array}
$$

see e.g. $[26,12]$. The concrete connection is

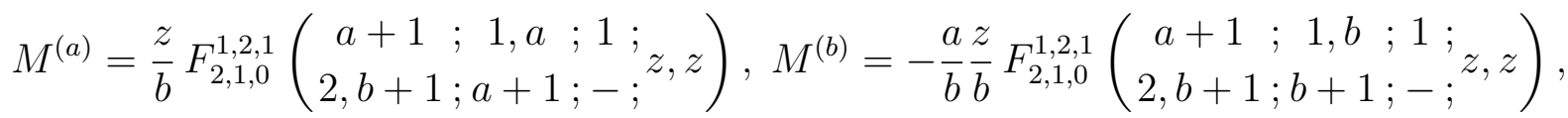

where the empty product indicated by the the solid horizontal line is interpreted to be unity. Employing this formula and proceeding similarly as in Section 5.1, one then obtains

$$
\begin{aligned}
\mathbb{E}\left[\tau_{x}\right] & =-\left.\frac{d}{d \delta} m_{\delta}(x)\right|_{\delta=0}=\frac{1+e^{z(0)} \gamma\left(1+\frac{\lambda}{q}, z(0)\right) z(0)^{-\frac{\lambda}{q}}}{\lambda} \\
& +\frac{e^{z(0)} z(0) F_{1,1,0}^{0,2,1}\left(\begin{array}{c}
-; 1,1+\frac{\lambda}{q} ; 1 ; \\
2, ; 2+\frac{\lambda}{q} ;-;
\end{array} ;(0),-z(0)\right)-e^{z(x)} z(x) F_{1,1,0}^{0,2,1}\left(\begin{array}{c}
\left.-; 1,1+\frac{\lambda}{q} ; 1 ;-z(x),-z(x)\right) \\
2, ; 2+\frac{\lambda}{q} ;-;-z
\end{array}\right.}{q+\lambda}
\end{aligned}
$$


The equivalency of this expression with (31) follows from the reduction formula (cf. [20])

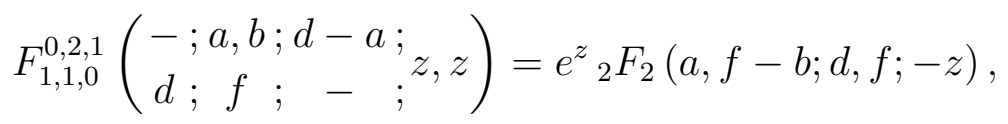

with $a=1, b=1+\frac{\lambda}{q}, d=2$ and $f=2+\frac{\lambda}{q}$.

\section{A probabilistic ARgUMEnT}

Inspired by Avanzi \& Wong [6], we also present here a probabilistic argument to connect the function $V(x)$ of Section 3 with the Laplace transform of the time to ruin in the previous section. To that end, consider first a surplus process of the form (4), but let it continue after ruin (i.e. whenever $X_{t}<-\frac{\beta}{q}$, negative dividends are paid, which could be interpreted as capital injections). ${ }^{2}$ Denote with

$$
\bar{V}(x):=\mathbb{E}_{x}\left[\int_{0}^{\infty} e^{-\delta t}\left(q X_{t}+\beta\right) d t\right]
$$

the respective expected discounted dividend payments (or, more precisely, the difference between expected discounted dividend payments and expected discounted amount of such capital injections).

Proposition 6.1. For $x \geq 0$,

$$
\bar{V}(x)=\frac{c-\lambda \mu}{\delta}+\frac{x-\frac{c-\beta-\lambda \mu}{q}}{1+\delta / q} .
$$

Proof.

$$
\begin{aligned}
\bar{V}(x) & =\mathbb{E}_{x}\left[\int_{0}^{\infty} e^{-\delta t}\left[q\left(\left(x-\frac{c-\beta}{q}\right) e^{-q t}+\frac{c-\beta}{q}-\int_{0}^{t} e^{-q(t-u)} d S_{u}\right)+\beta\right] d t\right] \\
& =\frac{q x}{q+\delta}+\frac{(c-\beta) q}{\delta(q+\delta)}+\frac{\beta}{\delta}-\mathbb{E}\left[\int_{0}^{\infty} q e^{q u} \int_{u}^{\infty} e^{-(q+\delta) t} d t d S_{u}\right] \\
& =\frac{q x}{q+\delta}+\frac{(c-\beta) q}{\delta(q+\delta)}+\frac{\beta}{\delta}-\frac{q}{q+\delta} \mathbb{E}\left[\sum_{i=1}^{\infty} e^{-\delta T_{i}} Y_{i}\right]
\end{aligned}
$$

where the last equality follows from $\lim _{t \rightarrow \infty} N_{t}=\infty$ a.s. Since the $i$-th claim arrival time $T_{i}$ in the Poisson model is independent of $Y_{i}$ and $\Gamma(i, \lambda)$-distributed, we then have

$$
\bar{V}(x)=\frac{q x}{q+\delta}+\frac{(c-\beta-\lambda \mu) q}{\delta(q+\delta)}+\frac{\beta}{\delta}, \quad x \geq 0 .
$$

\footnotetext{
${ }^{2}$ In fact, there is some methodological link to a calculation in Tichy [30], where for a horizontal dividend barrier an explicit calculation for $V$ without ruin was given.
} 
Due to the strong Markov property of $X_{t}$, we can now deduce

$$
V(x)=\bar{V}(x)-\mathbb{E}\left[e^{-\delta \tau_{x}} \bar{V}\left(X_{\tau_{x}}\right)\right], \quad x \geq 0 .
$$

For exponentially distributed claims with mean $\mu=1 / \alpha$, the lack-of-memory property implies that the deficit at $t=\tau_{x}$ is again exponentially distributed and independent of the time of ruin. This leads to

$$
V(x)=\bar{V}(x)-\mathbb{E}\left[e^{-\delta \tau_{x}}\right] \bar{V}\left(-\frac{1}{\alpha}\right) .
$$

Combining Proposition 6.1 with the expression for the Laplace transform of the time of ruin derived in (28) then again leads to the formula given in Proposition 3.3. In particular, this approach gives a complementary probabilistic interpretation of the particular solution $V_{p}(X)=\bar{V}(x)$ in Section 3 as well as the relation between the time of ruin and amount of dividend payments, in a certain sense akin to the dividends-penalty identity of Gerber et al. [15] in the model with horizontal dividend barrier.

\section{Numerical Illustrations}

7.1. General considerations. In this section we analyze the effects of the affine dividend strategy numerically. In particular, we are interested in the influence of the parameters $q$ and $\beta$ on the expected discounted dividend payments. Assume that $\alpha=1 / 3, \lambda=1, c=3.5$ and $\delta=0.05$. With each pair $(q, \beta) \in \mathbb{R}_{+} \times[0, c]$, we associate $V(x ; q, \beta):=V(x)$. Table 7.1 shows the influence of $q$ on $V(x)$ for $\beta=1.5$. We observe that $V(x ; q, 1.5)$ increases in $q$ up to a certain value and decreases thereafter. This demonstrates the compromise between paying larger amounts early (which is preferable due to discounting) and maintaining a longer survival in order to receive more payments later, i.e. too large proportions $q$ (in addition to the constant rate $\beta$ ) reduce the lifetime of the process too much. One observes that this turning point appears for larger values of $q$ the larger the initial surplus value $x$ is (and for $x=20$, this turning point is not yet visible for the depicted range of $q$ ). Incidentally, for $x=0$ one sees that the choices $q=0.2$ and $q=0.5$ lead to roughly the same total expected dividend payouts (where for the larger $q$, more dividends are collected earlier and over a shorter portfolio's lifetime compared to the case with the smaller $q$, i.e. different time patterns of dividend payments here lead to the same aggregate payout in expectation). 


\begin{tabular}{||c||c|c|c|c|c|c||}
\hline \multicolumn{7}{||c||}{$V(x ; q, 1.5)$} \\
\hline \hline$x$ & $q=0.1$ & $q=0.2$ & $q=0.3$ & $q=0.5$ & $q=1$ & $q=10$ \\
\hline 0 & 3.385 & 3.403 & 3.406 & 3.403 & 3.389 & 3.344 \\
0.5 & 3.896 & 3.919 & 3.923 & 3.920 & 3.903 & 3.846 \\
1 & 4.401 & 4.430 & 4.436 & 4.433 & 4.414 & 4.349 \\
2 & 5.396 & 5.440 & 5.452 & 5.451 & 5.430 & 5.352 \\
3 & 6.371 & 6.435 & 6.454 & 6.459 & 6.440 & 6.354 \\
4 & 7.327 & 7.415 & 7.445 & 7.458 & 7.443 & 7.356 \\
5 & 8.268 & 8.384 & 8.426 & 8.450 & 8.442 & 8.356 \\
10 & 12.763 & 13.079 & 13.213 & 13.321 & 13.381 & 13.352 \\
20 & 21.052 & 22.007 & 22.433 & 22.818 & 23.117 & 23.324 \\
\hline
\end{tabular}

TABLE 1. Expected present value of dividends for different rates $q$ with $\alpha=1 / 3, \lambda=1, c=3.5, \beta=1.5$ and $\delta=0.05$.

Next, let us consider the effect of $\beta$ on $V(x)$ for a given level of $q$. Table 2 illustrates a qualitatively similar pattern: the larger $x$ is, the higher constant rate $\beta$ can be afforded, and for $x \geq 10$, it is preferable to pay out the entire premium rate $c$ as dividends (in addition to the proportional payments).

\begin{tabular}{||c||c|c|c|c|c|c||}
\hline \multicolumn{7}{||c||}{$V(x ; 0.3, \beta)$} \\
\hline \hline$x$ & $\beta=0$ & $\beta=0.5$ & $\beta=1$ & $\beta=2$ & $\beta=3$ & $\beta=3.5$ \\
\hline 0 & 3.354 & 3.394 & 3.409 & 3.394 & 3.355 & 3.333 \\
0.5 & 3.855 & 3.903 & 3.922 & 3.913 & 3.876 & 3.854 \\
1 & 4.352 & 4.407 & 4.432 & 4.428 & 4.393 & 4.372 \\
2 & 5.336 & 5.405 & 5.440 & 5.449 & 5.419 & 5.399 \\
3 & 6.307 & 6.390 & 6.435 & 6.457 & 6.434 & 6.415 \\
4 & 7.267 & 7.363 & 7.418 & 7.453 & 7.438 & 7.422 \\
5 & 8.217 & 8.326 & 8.391 & 8.440 & 8.433 & 8.420 \\
10 & 12.863 & 13.028 & 13.139 & 13.258 & 13.298 & 13.302 \\
20 & 21.860 & 22.108 & 22.294 & 22.537 & 22.675 & 22.721 \\
\hline
\end{tabular}

TABLE 2. Expected present value of dividends for different rates $\beta$ with $\alpha=1 / 3, \lambda=1, c=3.5, q=0.3$ and $\delta=0.05$.

In order to better understand the contribution of the proportional rate $q$ and the constant rate $\beta$ to the overall value of $V(x)$, we now decompose (6) as $V(x):=$ 
$V_{q}(x)+V_{\beta}(x)$, where

$$
V_{q}(x):=\mathbb{E}_{x}\left[\int_{0}^{\tau_{x}} e^{-\delta t} q X_{t} d t\right], \quad \text { and } \quad V_{\beta}(x):=\mathbb{E}_{x}\left[\int_{0}^{\tau_{x}} e^{-\delta t} \beta d t\right] .
$$

Along the same line of arguments as in Section 3 one can then derive

$$
V_{q}(x)=A_{q} M\left(\frac{\delta}{q}, 1+\frac{\lambda+\delta}{q}, z(x)\right)+V_{q_{p}}(x), \quad x \geq 0,
$$

and

$$
V_{\beta}(x)=A_{\beta} M\left(\frac{\delta}{q}, 1+\frac{\lambda+\delta}{q}, z(x)\right)+V_{q_{p}}(x), \quad x \geq 0,
$$

for respective constants $A_{q}$ and $A_{\beta}$. We now proceed with an example to discuss the influence of the expected claim size $1 / \alpha$ on the relative contribution of (32) and (33) to the total expected dividend payouts (6). Consider the following constellation of parameters: $\lambda=1, c=5, q=0.5, \beta=1$ and $\delta=0.05$ so that the dividend rate at time $t$ is given by $0.5 X_{t}+1$. Hence, the linear term $q X_{t}$ constitutes the dominant term in the dividend rate if $X_{t}>2$. Figure 2 displays the ratio $V_{q}(x) / V(x)$ for $\alpha=1 / 3$ (solid line) and $\alpha=1 / 4$ (dashed line). First, we observe that the ratio $V_{q}(x) / V(x)$ is increasing in $x$. This is in line with intuition since for larger initial capital $x$, the proportion of dividends from the linear term is larger; the concrete value of that ratio, however, reflects the occupation time of the various levels of the process. This is also illustrated by the different response of the ratio $V_{q}(x) / V(x)$ on changing the average claim size, depending on the range of $x$.

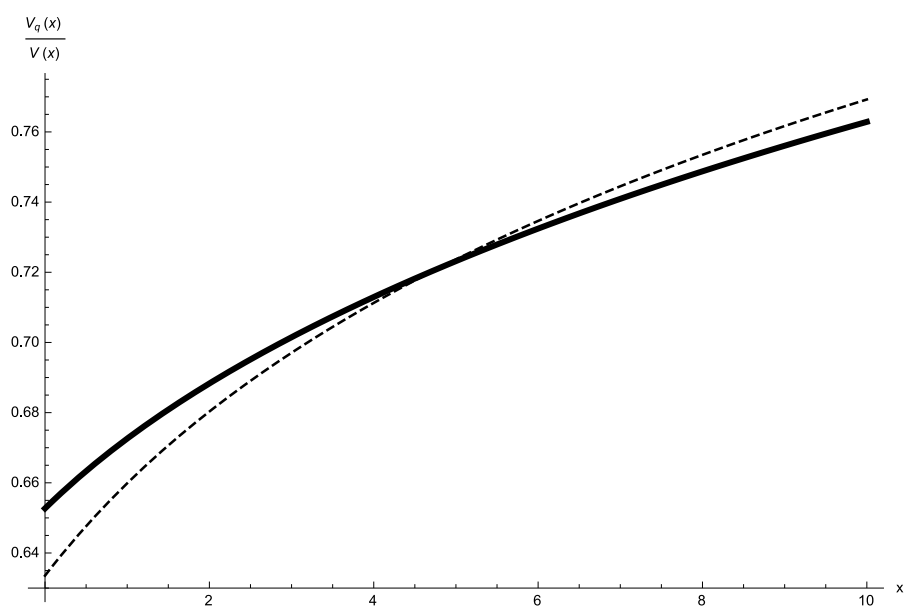

Figure 2: Ratio $V_{q}(x) / V(x)$ for $\alpha=1 / 3$ (solid line) and $\alpha=1 / 4$ (dashed line) 
7.2. Optimal parameters. In a next step, it is natural to ask which combination of parameters $q$ and $\beta$ maximizes the expected present value of dividends until ruin. Let $\Theta=\{(q, \beta): q>0, \beta \in[0, c]\}$. With each pair $(q, \beta) \in \Theta$, we associate $V(x ; q, \beta):=V(x)$. The optimization problem then consists in finding a pair $\left(q^{*}, \beta^{*}\right)$ such that

$$
V\left(x ; q^{*}, \beta^{*}\right)=\max _{(q, \beta) \in \Theta} V(x ; q, \beta),
$$

for a given initial capital $x \geq 0$. In view of (17), such an optimization problem has to be approached numerically (here we used respectives routines in Mathematica). Table 3 displays the optimal parameters and resulting optimal dividend values for the case $\alpha=1 / 3, \lambda=1, c=3.5$ and $\delta=0.05$ and different initial capital values $x$. For illustration purposes we also depict the corresponding values for $\delta=0.07$ in parentheses. One can observe that $q^{*}(x)$ increases in $x$ (note that $q^{*}(x)$ is chosen as a function of initial capital $x$, but by construction then kept fixed throughout the life-time of the process, i.e. not a function of current surplus value). Furthermore, we always have $\beta^{*}(x)=0$, i.e. a constant dividend rate does not contribute favorably to the compromise between profitability and length (lifetime) of the payments (this is also the case for other parameter values in numerical experiments). An interpretation of the latter is that since such a constant rate would be applied at all capital levels, the survival when close to ruin is more important than the payment of immediate dividends, which is somewhat in line with the philosophy behind dividend barrier strategies, cf. Section 7.3. For the higher discount rate $\delta=0.07, q^{*}(x)$ changes drastically and even becomes infinity for large $x$, mimicking lump sum payments of a barrier strategy with barrier at level zero. Note from (4) that for $\left(c-\beta^{*}(x)\right) / q^{*}(x) \geq$ $x$, the drift of the process $X_{t}$ will never be positive, cf. Figure 3 .

\begin{tabular}{||c||c||c|c||}
\hline$x$ & $V\left(x ; q^{*}, \beta^{*}\right)$ & $q^{*}(x)$ & $\beta^{*}(x)$ \\
\hline 0 & $3.426(3.279)$ & $0.751(3.789)$ & $0.000(0.000)$ \\
0.5 & $3.939(3.780)$ & $0.756(3.871)$ & $0.000(0.000)$ \\
1 & $4.449(4.280)$ & $0.768(4.088)$ & $0.000(0.000)$ \\
2 & $5.461(5.279)$ & $0.806(4.896)$ & $0.000(0.000)$ \\
3 & $6.466(6.276)$ & $0.860(6.413)$ & $0.000(0.000)$ \\
4 & $7.465(7.274)$ & $0.927(9.502)$ & $0.000(0.000)$ \\
5 & $8.460(8.272)$ & $1.008(18.227)$ & $0.000(0.000)$ \\
10 & $13.406(13.271)$ & $1.719(\infty)$ & $0.000(0.000)$ \\
20 & $23.334(23.271)$ & $31.623(\infty)$ & $0.000(0.000)$ \\
\hline
\end{tabular}

TABLE 3. Maximal expected present value of dividends and optimal pairs $\left(q^{*}, \beta^{*}\right)$ for $\alpha=1 / 3, \lambda=1, c=3.5$ and $\delta=0.05(\delta=0.07)$. 


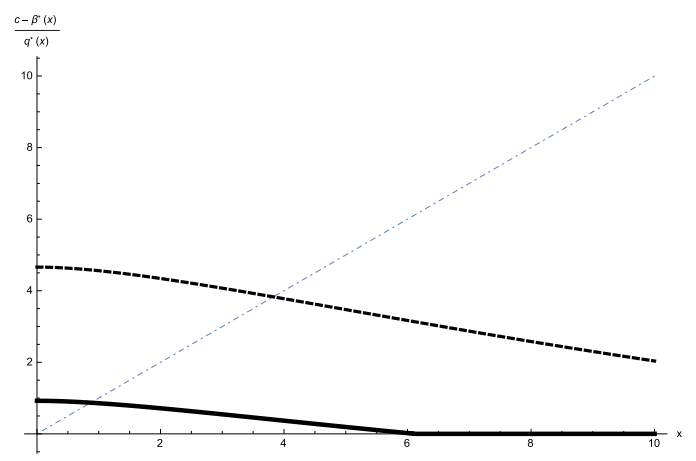

Figure 3: $\frac{c-\beta^{*}(x)}{q^{*}(x)}$ for $\delta=0.05$ (dashed line) and $\delta=0.07$ (solid line).

7.3. Comparison with the optimal barrier strategy. From classical results one knows that the optimal dividend strategy in a compound Poisson model with exponential claims is a barrier strategy (cf. Gerber [14]), if the sole criterion is the profitability. It is hence instructive to compare our optimal expected dividend payouts $V\left(x ; q^{*}, \beta^{*}\right)$ according to the affine dividend strategy with $V_{b^{*}}(x)$, the one under the optimal barrier strategy $b^{*}$. Recall that

$$
V_{b^{*}}(x)= \begin{cases}\frac{h(x)}{h^{\prime}\left(b^{*}\right)}, & 0 \leq x \leq b^{*} \\ x-b+\frac{h\left(b^{*}\right)}{h^{\prime}\left(b^{*}\right)}, & x>b^{*}\end{cases}
$$

where $h(u)=(r+\alpha) e^{r x}-(s+\alpha) e^{s x}, r>0$ and $s<0$ are the roots of the characteristic equation

$$
c \xi^{2}+(\alpha c-(\lambda+\delta)) \xi-\alpha \delta=0
$$

and

$$
b^{*}=\frac{1}{r-s} \ln \frac{s^{2}(s+\alpha)}{r^{2}(r+\alpha)}
$$

Table 4 compares the resulting $V_{b^{*}}(x)$ to the dividend payout of the optimal affine strategy $V\left(x ; q^{*}, \beta^{*}\right)$ for the case $\alpha=1 / 3, \lambda=1, c=3.5$ and $\delta=0.05$ (in which case $\left.b^{*}=3.26\right)$. Knowing that the barrier strategy is optimal among all admissible strategies, it is quite remarkable to observe how close one gets to this optimal value $V_{b^{*}}(x)$ by the best affine strategy. 


\begin{tabular}{||c||c||c|c|c||}
\hline \hline$x$ & $V_{b^{*}}(x)$ & $V\left(x ; q^{*}, \beta^{*}\right)$ & $q^{*}(x)$ & $\beta^{*}(x)$ \\
\hline 0 & 3.437 & 3.426 & 0.751 & 0 \\
$0.5 b^{*}$ & 5.232 & 5.223 & 0.795 & 0 \\
$b^{*}$ & 7.000 & 6.994 & 0.893 & 0 \\
$1.5 b^{*}$ & 8.764 & 8.749 & 1.034 & 0 \\
$2 b^{*}$ & 10.527 & 10.496 & 1.226 & 0 \\
$3 b^{*}$ & 14.055 & 13.981 & 1.854 & 0 \\
$5 b^{*}$ & 21.110 & 20.977 & 7.668 & 0 \\
\hline
\end{tabular}

TABlE 4. Comparison of the expected present value of dividends under affine and barrier dividend strategies for initial capitals $x$ with $\alpha=1 / 3, \lambda=1, c=3.5, q=0.3$ and $\delta=0.05$.

A next question in this context is then how sensitive the performance of the affine dividend strategy is when varying $(q, \beta) \in \Theta$. To get an impression on that, Figure 4 depicts the contour lines $\left\{(q, \beta) \in \Theta: V(x ; q, \beta)=a V_{b^{*}}(x)\right\}$, i.e. those parameter values for which we achieve a certain percentage $a$ of the optimal dividend barrier strategy. Here $x=10$ and $a \in[0.98,0.9942]$. The red area for instance consists of all pairs $(q, \beta)$ leading to at least $99.4 \%$ of $V_{b^{*}}(10)$. The size of that area is quite remarkable, showing that one can achieve quite convincing performance for a variety of $(q, \beta)$-values. The pair $(q, \beta)=(1.719,0)$ maximizes $V(10 ; q, \beta)$ and yields $99.5 \%$ of $V_{b^{*}}(10)$. One also sees that the gradient becomes larger as one moves towards smaller $q$-values indicating that $V(10 ; q, \beta)$ is sensitive to changes in $q$ for smaller $q$.

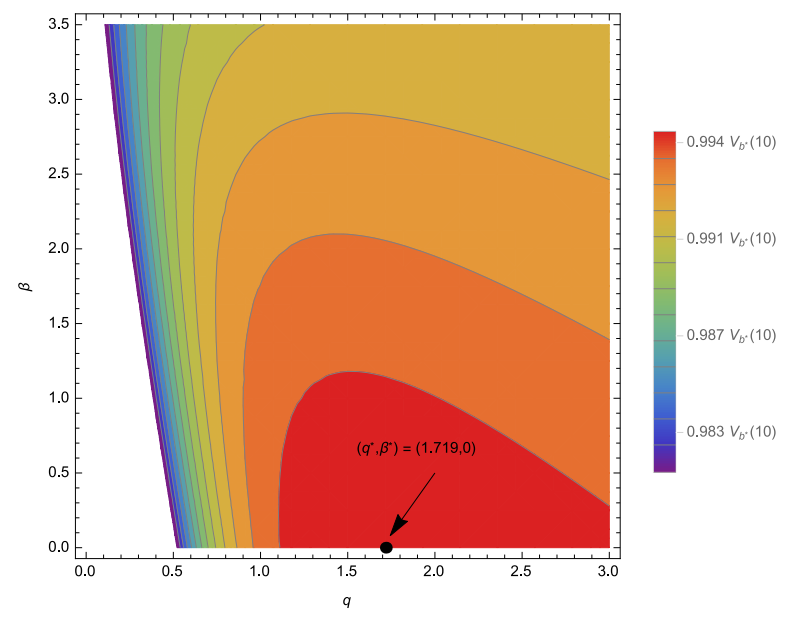

Figure 4: Contour lines for $x=10, \alpha=1 / 3, \lambda=1, c=3.5, q=0.3$ and $\delta=0.05$. 
7.4. Dividend payments versus expected ruin time. Since one motivation to introduce an affine dividend strategy was the increased lifetime of the process, and since we have seen in the previous section that the performance of this strategy gets quite close to the one of optimal barrier strategies, it is now interesting to see to what extent the expected ruin time is improved using such an affine dividend strategy.

Let $T_{x}^{b}$ the time to ruin with a barrier at level $b$. The Laplace transform of $T_{x}^{b}$ for exponentially distributed claim amounts in the compound Poisson model is then given by

$$
\mathbb{E}\left[e^{-\delta T_{x}^{b}}\right]= \begin{cases}\frac{(s+\alpha)(r+\alpha)}{\alpha}\left[\frac{r e^{r b} e^{s x}-s e^{s b} e^{r x}}{r(r+\alpha) e^{r b}-s(s+\alpha) e^{s b}}\right] & 0 \leq x \leq b, \\ \frac{(s+\alpha)(r+\alpha)}{\alpha}\left[\frac{e^{(r+s) b}(r-s)}{r(r+\alpha) e^{r b}-s(s+\alpha) e^{s b}}\right] & x>b,\end{cases}
$$

see e.g. [17, Equ.6.3]). We then have

$$
\mathbb{E}\left[T_{x}^{b}\right]=-\left.\frac{d}{d \delta} \mathbb{E}\left[e^{-\delta T_{x}^{b}}\right]\right|_{\delta=0} .
$$

Let $\mathbb{E}\left[\tau_{x ; q, \beta}\right]$ be the expected ruin time under the affine dividend strategy $(q, \beta) \in \Theta$ (cf. Proposition 5.2) and consider the following constrained optimization problem:

$$
\begin{array}{ll}
\max _{(q, \beta) \in \Theta} & \mathbb{E}\left[\tau_{x ; q, \beta}\right] \\
\text { subject to } & V(x ; q, \beta)=a V_{b^{*}}(x),
\end{array}
$$

where $a \in(0,1)$. Note that the resulting optimal values $q^{*}$ are not the same as the ones in the previous section, whereas $\beta^{*}$ turns out to be again always zero. Figure 5 depicts the ratio $\mathbb{E}\left[\tau_{x ; q^{*}, \beta^{*}}\right] / \mathbb{E}\left[T_{x}^{b^{*}}\right]$ for different initial capital values $x$ as function of the performance factor $a$, for the same parameters $\alpha=1 / 3, \lambda=1, c=3.5$ and $\delta=0.05$. To match a higher required performance level $a$, one has to select larger values in the set $\Theta$, which causes a reduction of the expected time to ruin $\mathbb{E}\left[\tau_{x ; q^{*}, \beta^{*}}\right]$. Hence, the ratio $\mathbb{E}\left[\tau_{x ; q^{*}, \beta^{*}}\right] / V_{b^{*}}(x)$ is monotone decreasing in $a$. For $x=2$, we have that for $a=0.95$, selecting the best pair $\left(q^{*}, \beta^{*}\right)$ roughly doubles the expected life time of the portfolio, whilst for $a=0.99$, the improvement factor is still 1.33. For $x=4$, it is worth noticing that under the horizontal dividend strategy (with $\left.b^{*}=3.257\right)$, an immediate dividend payment occurs, leading to a reduced expected ruin time $\mathbb{E}\left[T_{b^{*}}^{b^{*}}\right]$. Here the affine dividend strategy then compares even more favorably in terms of lifetime of the process. However, this trend is not preserved for ever higher values of $x$. For illustrative purposes, we consider $x=50$, where clearly there is a considerable initial dividend payment under the optimal barrier strategy, contributing to a major extent to the overall value $V_{b^{*}}(50)$. Matching this performance under an affine dividend strategy for some large factor $a$, say 0.99 , requires to increase $\left(q^{*}, \beta^{*}\right)$ to an extent that the ratio $\mathbb{E}\left[\tau_{x ; q^{*}, \beta^{*}}\right] / \mathbb{E}\left[T_{x}^{b}\right]$ is then even below 
1 (see dotted line at $a=0.99$ ).

Figure 6 depicts the resulting maximizer $q^{*}$ for $x=2$ (solid line), $x=4$ (dashed line) and $x=50$ (dotted line) as a function of $a$. For $x=2$ and $x=4$, the choice of $q^{*}$ is almost identical. However, for $x=50$, a significant non-linear increase of $q^{*}$ is needed for higher values of $a$ to make up for the large initial lump sum payment under the optimal barrier strategy.

Altogether, one sees that a suitably chosen affine dividend strategy can lead to almost as large values for the expected discounted dividend payments, while leading to considerably improved safety, measured in terms of expected ruin time of the portfolio. Note that the chosen numerical values of the discount rate $\delta$ are quite high, and smaller values of $\delta$ can lead to an even better performance of the affine strategy relative to the optimal barrier strategy.

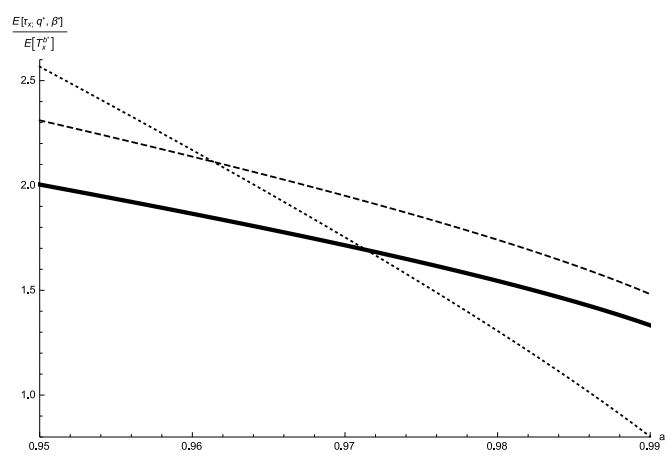

Figure 5: Ratio $\mathbb{E}\left[\tau_{x ; q^{*}, \beta^{*}}\right] / \mathbb{E}\left[T_{x}^{b^{*}}\right]$ for $x=2$ (solid line), $x=4$ (dashed line) and $x=50$ (dotted line).

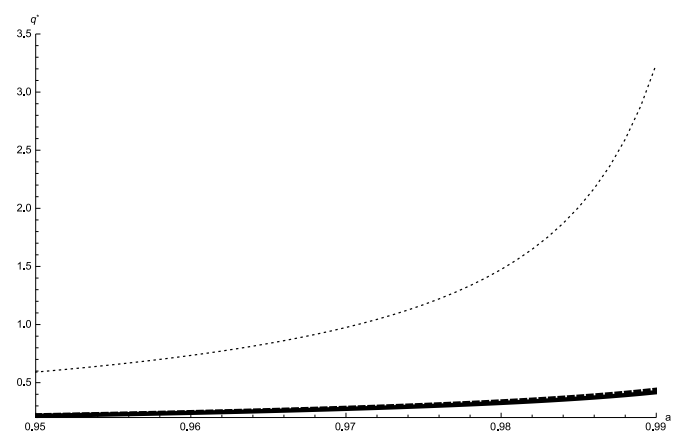

Figure 6: Maximizer $q^{*}$ for $x=2$ (solid line), $x=4$ (dashed line) and $x=50$ (dotted line). 


\section{REFERENCES}

[1] M. Abramowitz and I. A. Stegun. Handbook of mathematical functions with formulas, graphs, and mathematical tables, volume 55 of National Bureau of Standards Applied Mathematics Series. 1964.

[2] H. Albrecher, R. Kainhofer, and R. F. Tichy. Simulation methods in ruin models with non-linear dividend barriers. Math. Comput. Simulation, 62(3-6):277-287, 2003. 3rd IMACS Seminar on Monte Carlo Methods-MCM 2001 (Salzburg).

[3] H. Albrecher and S. Thonhauser. Optimality results for dividend problems in insurance. Rev. R. Acad. Cienc. Exactas Fís. Nat. Ser. A Math. RACSAM, 103(2):295-320, 2009.

[4] S. Asmussen and H. Albrecher. Ruin probabilities. Advanced Series on Statistical Science \& Applied Probability, 14. World Scientific Publishing Co. Pte. Ltd., Hackensack, NJ, second edition, 2010.

[5] B. Avanzi. Strategies for dividend distribution: a review. N. Am. Actuar. J., 13(2):217-251, 2009.

[6] B. Avanzi and B. Wong. On a mean reverting dividend strategy with Brownian motion. Insurance Math. Econom., 51(2):229-238, 2012.

[7] P. Azcue and N. Muler. Stochastic Optimization in Insurance: a Dynamic Programming Approach. Springer Briefs in Quantitative Finance. Springer, 2014.

[8] A. Brav, J. R. Graham, C. R. Harvey, and R. Michaely. Payout policy in the 21st century. Journal of Financial Economics, 77(3):483-527, 2005.

[9] R. Cont and P. Tankov. Financial modelling with jump processes. Chapman \& Hall/CRC Financial Mathematics Series. Chapman \& Hall/CRC, Boca Raton, FL, 2004.

[10] D. Cvijović. Closed-form summations of certain hypergeometric-type series containing the digamma function. Journal of Physics A: Mathematical and Theoretical, 41(45):455205, 2008.

[11] B. De Finetti. Su unimpostazione alternativa della teoria collettiva del rischio. In Transactions of the XVth International Congress of Actuaries, volume 2, pages 433-443, 1957.

[12] H. Exton. Multiple hypergeometric functions and applications. Wiley, Chichester, 1976.

[13] H. U. Gerber.

[14] H.-U. Gerber. Entscheidungskriterien für den zusammengesetzten Poisson-Prozess. PhD thesis, 1969.

[15] H. U. Gerber, S. Lin, and H. Yang. A note on the dividends-penalty identity and the optimal dividend barrier. ASTIN Bulletin, 36(2):489-503, 2006.

[16] M. J. Gordon. Dividends, earnings and stock prices. Review of Economics and Statistics, 41:99$105,1959$.

[17] X. S. Lin, G. E. Willmot, and S. Drekic. The classical risk model with a constant dividend barrier: analysis of the Gerber-Shiu discounted penalty function. Insurance Math. Econom., $33(3): 551-566,2003$.

[18] J. Lintner. Distribution of incomes of corporations among dividends, retained earnings, and taxes. The American Economic Review, 46(2):97-113, 1956.

[19] R. L. Loeffen and J.-F. Renaud. De Finetti's optimal dividends problem with an affine penalty function at ruin. Insurance Math. Econom., 46(1):98-108, 2010.

[20] A. R. Miller. Summations for certain series containing the digamma function. J. Phys. A, 39(12):3011-3020, 2006. 
[21] F. W. J. Olver, D. W. Lozier, R. F. Boisvert, and C. W. Clark, editors. NIST Handbook of Mathematical Functions. U.S. Department of Commerce, National Institute of Standards and Technology, Washington, DC; Cambridge University Press, Cambridge, 2010.

[22] M. Parlar. Use of stochastic control theory to model a forest management system. Applied mathematical modelling, 9(2):125-130, 1985.

[23] K. Sato. Lévy processes and infinitely divisible distributions. Cambridge University Press, Cambridge, 2013.

[24] H. Schmidli. Stochastic control in insurance. Probability and its Applications (New York). Springer-Verlag London, Ltd., London, 2008.

[25] W. Schoutens. Lévy Processes in Finance. Wiley, New York, 2003.

[26] H. M. Srivastava and P. W. Karlsson. Multiple Gaussian hypergeometric series. Wiley, Chichester, 1985.

[27] M. Steffensen. Quadratic optimization of life and pension insurance payments. Astin Bulletin, $36(01): 245-267,2006$.

[28] S. Thonhauser and H. Albrecher. Dividend maximization under consideration of the time value of ruin. Insurance Math. Econom., 41(1):163-184, 2007.

[29] R. F. Tichy. Über eine zahlentheoretische Methode zur numerischen Integration und zur Behandlung von Integralgleichungen. Österreich. Akad. Wiss. Math.-Natur. Kl. Sitzungsber. II, 193(4-7):329-358, 1984.

[30] R. F. Tichy. Bemerkung zu einem versicherungsmathematischen Modell. Mitt. Verein. Schweiz. Versicherungsmath., (2):237-241, 1987.

E-mail address: hansjoerg.albrecher@unil.ch

University of Lausanne and Swiss Finance Institute, Quartier UNIL-Dorigny, BÂtiment

Extranef, 1015 Lausanne, Switzerland

E-mail address: arian.cani@unil.ch

University of Lausanne, Quartier UniL-Dorigny, BÂtiment Extranef, 1015 LaUSANNE, SWITZERLAND 